\title{
Operational Resource Theory of Continuous-Variable Nonclassicality
}

\author{
Benjamin Yadin, ${ }^{1,5,}$ Felix C. Binder, ${ }^{2,3}$ Jayne Thompson, ${ }^{4}$ Varun Narasimhachar, ${ }^{2,3}$ Mile Gu, ${ }^{2,3,4, \dagger}$ and M. S. Kim ${ }^{1}$ \\ ${ }^{1}$ QOLS, Blackett Laboratory, Imperial College London, London SW7 2AZ, United Kingdom \\ ${ }^{2}$ School of Physical and Mathematical Sciences, Nanyang Technological University, \\ 637371 Singapore, Singapore \\ ${ }^{3}$ Complexity Institute, Nanyang Technological University, 637335 Singapore, Singapore \\ ${ }^{4}$ Centre for Quantum Technologies, National University of Singapore, \\ 3 Science Drive 2, 117543 Singapore, Singapore \\ ${ }^{5}$ Clarendon Laboratory, Department of Physics, University of Oxford, \\ Parks Road, Oxford OX1 3PU, United Kingdom
}

(Received 16 May 2018; revised manuscript received 10 October 2018; published 3 December 2018)

\begin{abstract}
Genuinely quantum states of a harmonic oscillator may be distinguished from their classical counterparts by the Glauber-Sudarshan $P$ representation-a state lacking a positive $P$ function is said to be nonclassical. In this paper, we propose a general operational framework for studying nonclassicality as a resource in networks of passive linear elements and measurements with feed forward. Within this setting, we define new measures of nonclassicality based on the quantum fluctuations of quadratures, as well as the quantum Fisher information of quadrature displacements. These measures lead to fundamental constraints on the manipulation of nonclassicality, especially its concentration into subsystems, that apply to generic multimode non-Gaussian states. Special cases of our framework include no-go results in the concentration of squeezing and a complete hierarchy of nonclassicality for single-mode Gaussian states.
\end{abstract}

DOI: 10.1103/PhysRevX.8.041038

\section{INTRODUCTION}

Continuous-variable quantum-optical systems exhibit numerous operational advantages over their discrete counterparts, including the unconditional generation of entanglement and relative resilience of such nonclassical states to photon loss. They have thus played an important role in diverse quantum technologies, spanning computing [1-4], communication [5-7], and metrology [8,9]. Indeed, the reliable creation of entanglement has allowed recent synthesis of ultralarge entangled clusters that would be difficult to achieve in any other regime [10].

This divergence in what information processing tasks are considered operationally difficult also motivates different perspectives on nonclassicality. In discrete variables, nonclassicality is often characterized in terms of coherence and entanglement-the former to capture the difficulty of creating a quantum superposition of states in some designated classical basis (such as energy eigenstates) and

\footnotetext{
*benjamin.yadin@gmail.com

†mgu@quantumcomplexity.org
}

Published by the American Physical Society under the terms of the Creative Commons Attribution 4.0 International license. Further distribution of this work must maintain attribution to the author(s) and the published article's title, journal citation, and DOI.
Subject Areas: Optics, Quantum Physics,

Quantum Information the latter to characterize the difficulty of entangling two quantum systems. In contrast, in continuous variables, coherent states of light are typically considered the most classical pure states $[11,12]$ owing to their ease of synthesis. Unlike the energy eigenstates, coherent states are not mutually orthogonal and represent a superposition of different energies. A state is considered nonclassical when it is not a probabilistic mixture of coherent states [13]. Entanglement itself is considered secondary, as it is easily synthesized by passive linear optics (i.e., networks of beam splitters) once one has a source of nonclassical light $[14,15]$. This observation has motivated tailored means of witnessing and quantifying nonclassicality in the continuous-variable regime [16-26].

A full theoretical understanding of nonclassicality will likely take the form of a resource theory [27] — a mathematical formalism that has enjoyed notable success in the past two decades for describing the structure of entanglement $[28,29]$. A resource theory gives meaning to the question of how much of a quantity of interest is present in a given state. In doing so, it renders different states comparable. For instance, in the context of nonclassicality, one might ask if a given squeezed state or a Fock state $|n\rangle$ is more nonclassical-a question that can be clearly answered with the framework introduced below. Resource theories thus provide a set of criteria for determining whether a proposed quantity counts as a valid measure of the 
resource. They have been instrumental in understanding quantum reference frames [30], thermodynamics [31-34], coherence [35-37], contextuality [38], steering [39], and non-Gaussianity [40-43]. Resource-theoretic terminology in continuous variables has appeared in a number of recent works [44-52], but these ideas are still in their infancy.

The main contribution of this paper is an operationally motivated resource theory for continuous-variable nonclassicality where passive linear optics and measurement feed forward are considered operationally simple and thus "free." We show that this approach naturally leads to a novel quantum resource theory of phase-space variance that captures existing views of nonclassicality for both pure and mixed states. For pure states, phase-space variance can be analytically evaluated. For mixed states, we show it can be bounded from below by the quantum Fisher information (QFI) of quadrature displacements. Moreover, we prove that the QFI produces valid indicators of nonclassicality in their own right, having the same monotone behavior as phasespace variance. This result shows a quantitative relation between nonclassicality and performance in metrology. We use this framework to obtain powerful bounds on the concentration of nonclassicality that are applicable to general non-Gaussian multimode states. Specialization of these techniques to Gaussian states retrieves no-go theorems on the concentration of squeezing and full hierarchies of nonclassicality in the pure multimode and mixed single-mode cases.

\section{PASSIVE LINEAR OPTICS AND THE STRUCTURE OF THE RESOURCE THEORY}

We work with $n$ bosonic modes with corresponding creation and annihilation operators $a_{i}^{\dagger}, a_{i}$ for $i=1, \ldots, n$ satisfying the commutation relations $\left[a_{i}, a_{j}^{\dagger}\right]=\delta_{i, j}$. Quadratures operators are defined by $x_{i}=(1 / \sqrt{2})\left(a_{i}+a_{i}^{\dagger}\right)$, $p_{i}=[1 /(\sqrt{2} i)]\left(a_{i}-a_{i}^{\dagger}\right)$ and may be collected into the vector $\quad \boldsymbol{q}=\left(q_{1}, q_{2}, \ldots, q_{2 n-1}, q_{2 n}\right)=\left(x_{1}, p_{1}, \ldots, x_{n}, p_{n}\right)$ [53]. The canonical commutators are expressed via $\left[q_{s}, q_{t}\right]=i \Omega_{s t}$, where

$$
\Omega=\bigoplus_{i=1}^{n}\left(\begin{array}{cc}
0 & 1 \\
-1 & 0
\end{array}\right)
$$

is the symplectic structure. The quadrature corresponding to a general direction $\boldsymbol{r} \in \mathbb{R}^{2 n},|\boldsymbol{r}|=1$ in phase space is $\boldsymbol{r} \cdot \boldsymbol{q}$.

In a resource-theory viewpoint, one starts from the perspective that under certain physical conditions or constraints, particular quantum states and processes may be considered resources. The theory is specified by first deciding which sets of states and operations are free, meaning that they have no resource value. A sensible choice of free states are those that are easily prepared in the lab and similarly, free operations should be easily performed. This structure allows for the quantification of resources. A valid measure $M(\rho)$ of the resource value of a state $\rho$ must satisfy several criteria:

(i) $M(\rho) \geq 0$ and vanishes if and only if $\rho$ is a free state.

(ii) $M(\rho)$ is nonincreasing when $\rho$ undergoes a free operation.

(iii) Convexity, i.e., $M\left(\sum_{\mu} p_{\mu} \rho_{\mu}\right) \leq \sum_{\mu} p_{\mu} M\left(\rho_{\mu}\right)$ for any ensemble of states $\rho_{\mu}$ with probabilities $p_{\mu} \geq 0$, $\sum_{\mu} p_{\mu}=1$.

Property (i) is a natural requirement for ordering resources. Property (ii) expresses the fact that free operations cannot create more of the resource. This can be expressed in different versions, either for deterministic or probabilistic transformations. Any $M$ satisfying (ii) is called a monotone; we reserve the term measure for $M$ satisfying both (i) and (ii). Convexity is often desirable since probabilistic mixing is typically considered a free operation.

Here, building on the approach of Ref. [52], we choose the free states to coincide with the set $\mathcal{C}_{n}$ of classical states on $n$ modes consisting of convex mixtures of coherent states. In terms of the Glauber-Sudarshan $P$ function, we have

$$
\mathcal{C}_{n}:=\left\{\int d^{2 n} \boldsymbol{\alpha} P(\boldsymbol{\alpha})|\boldsymbol{\alpha}\rangle\langle\boldsymbol{\alpha}|| P(\boldsymbol{\alpha}) \geq 0\},\right.
$$

where $|\boldsymbol{\alpha}\rangle:=\left|\alpha_{1}\right\rangle, \ldots,\left|\alpha_{n}\right\rangle$ is a product of coherent states, and $\boldsymbol{\alpha}=\left(\alpha_{1}, \ldots, \alpha_{n}\right) \in \mathbb{C}^{n}$.

The choice of free operations is very important since it determines what it means for one state to be more nonclassical than another. In resource theories, generally it is not always possible to unambiguously say when one state is a more valuable resource than another-this may depend on the particular task being considered. However, we may say that if $\rho$ can be transformed into $\sigma$ via a free operation, then $\rho$ is at least as nonclassical as $\sigma$.

The set of free unitary operations is easily motivated to be passive linear (PL) unitaries, meaning all numberconserving $U$ such that $\left[U, \sum_{i=1}^{n} a_{i}^{\dagger} a_{i}\right]=0$ and displacements $D(\boldsymbol{\alpha}):=\prod_{i=1}^{n} e^{\alpha_{i} a_{i}^{\dagger}-\alpha_{i}^{*} a_{i}}$. These operations are the most general unitaries mapping the set of classical states $\mathcal{C}_{n}$ to itself [52], which is necessary for a consistent description of resources. Moreover, they are operationally free in optics, for instance, where all PL unitaries can be implemented with beam splitters and phase shifters [54], readily accessible optical elements [55]. Displacements are also routinely performed by mixing a state with a large-amplitude coherent state at a beam splitter [56]. By contrast, nonlinear operations are typically very weak and difficult to perform coherently $[57,58]$.

The relevant set of general (including nonunitary) free quantum operations is not so clear cut. In principle, one can take the largest set of classicality-preserving operations (CPOs) [23]. However, this is unsatisfactory, lacking a full mathematical characterization and physical motivation. 
Instead (following similar arguments for the resource theory of coherence [59-61]), we demand that all nonunitary free operations be implementable with free ancillas and free unitaries, plus measurements and discarding of modes. Therefore, we take the free operations to be all possible compositions of the following elements:

(1) The addition of uncorrelated classical ancilla modes

(2) PL unitaries and displacements

(3) Destructive measurements on any set of modes

(4) Conditioning on classical randomness and coarse graining

Note that arbitrary positive operator-valued measures (POVMs) are permissible as long as they are destructive, i.e., the measured modes are discarded afterwards. It is easily checked that these operations preserve classicality. (Note that there is no distinction between entangling and nonentangling measurements, since beam splitters are free.) In most applications, however, only a small subset of measurements may be feasible; e.g., when discussing Gaussian states in Sec. VI, we limit the discussion to Gaussian measurements. Nevertheless, all our results hold true with more restricted measurements. The final elements in the list simply allow for the conditioning of operations on a classical random number generator and the forgetting of classical information.

As we discuss in detail in Appendix A, our free operations can be described formally as quantum instruments [62]. A quantum instrument $\mathcal{I}$ is a (possibly infinite) collection of completely positive maps $\mathcal{I}=\left(\mathcal{A}_{1}, \mathcal{A}_{2}, \ldots\right)$ such that $\sum_{m} \mathcal{A}_{m}$ is trace preserving. Each $\mathcal{A}_{m}$ describes the operation resulting from a certain selected measurement outcome $m$. Coarse graining can be described by forming a new instrument $\mathcal{I}^{\prime}$ whose elements $\mathcal{A}_{m}^{\prime}$ are sums of distinct partitions of the $\mathcal{A}_{m}$. The most fine-grained description of an instrument consists of $\mathcal{A}_{m}$ which each have a single associated Kraus operator $K_{m}: \mathcal{A}_{m}(\rho)=K_{m} \rho K_{m}^{\dagger}$.

We distinguish between different classes of such operations (see Fig. 1).

(i) $\mathcal{P}_{0}$ is formed by adding a classical ancilla performing a PL unitary and tracing out a set of modes. An instrument $\mathcal{I} \in \mathcal{P}_{0}$ has a single element.

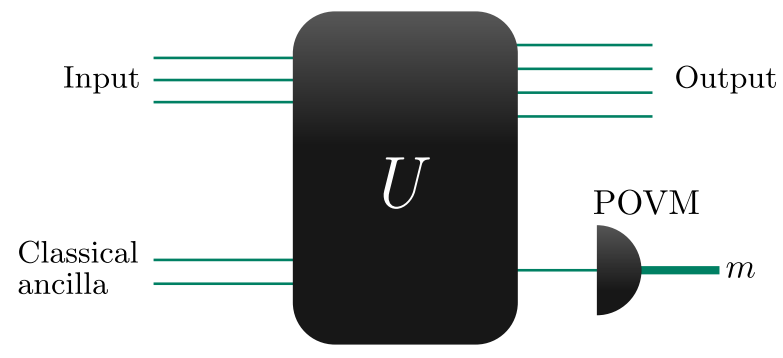

FIG. 1. An operation in the set $\mathcal{P}_{1}$ constructed with classical ancilla modes, a PL unitary $U$, and a POVM on a set of output modes. $\mathcal{P}_{\mathbb{N}}$ is constructed by repeated concatenation of $\mathcal{P}_{1}$ elements with feed forward depending on each measurement outcome $m . \mathcal{P}_{0}$ is the special case of $\mathcal{P}_{1}$ where the outcome $m$ is not recorded. (ii) $\mathcal{P}_{1}$ is the same as $\mathcal{P}_{0}$ but with a measurement first performed on the modes to be traced out.

(iii) $\mathcal{P}_{r}$ is the set of all protocols generated by repeated application of $\mathcal{P}_{1}$ with $r$ measurement rounds. Note that feed forward is permitted.

(iv) $\mathcal{P}_{\mathbb{N}}:=\bigcup_{r=1}^{\infty} \mathcal{P}_{r}$ is the set of all finite-length protocols.

Different numbers of input and output modes are permitted in general. In principle, any number of ancilla modes may be used at each stage. Despite this, we prove the following simplification (see Appendix B).

Lemma 1. The number of ancilla modes for an operation in $\mathcal{P}_{1}$ can be assumed to be no larger than the number of output modes.

Given the chosen sets of free operations, nonclassicality may be viewed generally as a resource in situations where one has access only to the free operations. For example, single-mode nonclassicality is a resource for creating entanglement via passive linear optics: There exists a free operation creating an entangled two-mode state from an input single-mode state $\rho$ if and only if $\rho$ is nonclassical $[14,22,63]$. Thus, by examining the structure of these operations, we expect to derive general statements about the utility of nonclassical states.

What kinds of operations are allowed by taking this free set rather than the maximal set of CPOs? The following result puts a strong constraint on the action that our free operations can have on coherent states; it proves that no process in $\mathcal{P}_{\mathbb{N}}$ is able to perform coherent amplification.

Theorem 1. (No free amplification.) Every operation in $\mathcal{P}_{\mathbb{N}}$ can be described by a set of Kraus operators $\left\{K_{m}\right\}$ satisfying

$$
K_{m}|\boldsymbol{\alpha}\rangle=c_{m}(\boldsymbol{\alpha})\left|M_{m} \boldsymbol{\alpha}+\boldsymbol{\delta}_{m}\right\rangle,
$$

where $\boldsymbol{\alpha} \in \mathbb{C}^{n}, c_{m}(\alpha) \in \mathbb{C}, \boldsymbol{\delta}_{m} \in \mathbb{C}^{n^{\prime}}$, and $M_{m} \in \mathbb{C}^{n^{\prime} \times n}$ has singular values of modulus $\leq 1$. Here, $n, n^{\prime}$ are the numbers of input and output modes, respectively.

(See Appendix C for full details.) Note that an operation is determined by its action on coherent states thanks to the $P$ representation. This demonstrates that every free operation can be viewed as a classical mixture of phasespace contractions (represented by $M_{m}$ ) and displacements (represented by $\boldsymbol{\delta}_{m}$ ).

Let us consider some examples of CPOs. Single-photon subtraction is a nondeterministic process mapping $\rho \rightarrow$ $a \rho a^{\dagger}$. It is free in our framework, being implementable in $\mathcal{P}_{1}$ using a vacuum ancilla, beam splitter, and single-photon detection $[64,65]$. On the other hand, take phase-insensitive noiseless linear amplification, which maps $|\alpha\rangle \mapsto|g \alpha\rangle$ with $|g|>1$ [66]. No deterministic process is able to do this, but it can be done probabilistically with a trace-decreasing map $\mathcal{E}$ such that $\mathcal{E}(|\alpha\rangle\langle\alpha|)=p|g \alpha\rangle\langle g \alpha|$, where $p \leq 1 /|g|^{2}$ [67]. Proposals to implement this utilize either singlephoton ancillas (which are nonclassical) [67] or nonlinear 
media [68]. In fact, Theorem 1 proves that the operation is not in $\mathcal{P}_{\mathbb{N}}$; i.e., no free implementation is possible.

Theorem 1 also has implications for the kinds of measures of nonclassicality that are expected in the resource theory. Every monotone under CPOs is necessarily a monotone under the subset $\mathcal{P}_{\mathbb{N}}$, but choosing a smaller set of free operations may in principle allow for more monotones. To gain some insight, consider a cat state $\left|\psi_{c}(\alpha)\right\rangle \propto|\alpha\rangle+|-\alpha\rangle$. Suppose we ask for a process taking $\left|\psi_{c}(\alpha)\right\rangle$ to $\left|\psi_{c}\left(\alpha^{\prime}\right)\right\rangle$. Every operation in $\mathcal{P}_{\mathbb{N}}$ is constrained to output $|\alpha|^{\prime} \leq|\alpha|$, while the above amplification process gives a CPO such that $|\alpha|^{\prime}>|\alpha|$ with nonzero probability.

Some known measures of nonclassicality will fail to capture this distinction. For example, one can define the distance to the set of classical states $\inf _{\sigma \in \mathcal{C}_{n}} D(\rho, \sigma)$ with some suitable distance measure $D[19,21]$. Taking the trace distance $D(\rho, \sigma)=\frac{1}{2} \operatorname{Tr}|\rho-\sigma|$, we obtain a monotone known as the "nonclassical distance" [19]. As shown in Ref. [69], for $|\alpha| \gg 1$, the dependence on $\alpha$ becomes negligible and saturates at $1 / 2$, so its monotonicity gives little information about the contractive behavior. This fact is essentially due to the near orthogonality of the branches in that limit. The same is true for the "entropic entanglement potential" defined as the entropy of entanglement created at a beam splitter, now saturating at 1 [22]. The nonclassical depth defined by Lee [20] (namely, the minimal amount of thermal noise that must be added to a state to make it classical), in fact, has its maximal value of 1 for all $\alpha$ [70]. Even more striking, the nonclassical distance is found to be decreasing with $|\alpha|$ in the case of an odd cat state $|\alpha\rangle-|-\alpha\rangle$ [69]. Hence, we may ask what kinds of measures truly capture the size of $|\alpha|$ in such superpositions.

\section{PHASE-SPACE QUANTUM VARIANCE}

We propose measures of nonclassicality which capture the separation in phase space of branches of a superposition. In the cat state example, the magnitude of $\alpha$ is an indicator of the macroscopic distinguishability of the branches of the superposition and, hence, of the quantum "macroscopicity" of the state $[71,72]$. This property is captured by the maximal variance over all quadratures $[73,74]$. For a single-mode pure state $|\psi\rangle$, we define

$$
\mathcal{V}_{1}(|\psi\rangle):=\max _{r \in \mathbb{R}^{2}:|\boldsymbol{r}|=1} V(|\psi\rangle, \boldsymbol{r} \cdot \boldsymbol{q})-\frac{1}{2},
$$

where $V(\rho, A):=\operatorname{Tr}\left[\rho A^{2}\right]-\operatorname{Tr}[\rho A]^{2}$ is the variance of observable $A$ in the state $\rho$. By definition, $\mathcal{V}_{1}$ is invariant under phase rotations. Moreover, it is a faithful witness of nonclassicality, vanishing if and only if $|\psi\rangle$ is classical. This result follows from the Heisenberg-Robertson uncertainty principle [75]: For any quadrature $x$ and its conjugate momentum $p, V(|\psi\rangle, x) V(|\psi\rangle, p) \geq 1 / 4$, so $\mathcal{V}_{1}(|\psi\rangle)=0$ is equivalent to the inequality being saturated with all variances equal to $1 / 2$; this means $|\psi\rangle$ must be Gaussian [76] and, moreover, a coherent state.

Alternatively, one can consider the total variance [77], which is related to another measure of macroscopicity [78]:

$$
\mathcal{W}_{1}(|\psi\rangle):=V(|\psi\rangle, x)+V(|\psi\rangle, p)-1 .
$$

This quantity is again non-negative and is found to be invariant under phase rotations. $\mathcal{W}_{1}$ also vanishes if and only if $|\psi\rangle$ is classical. This result follows from using the uncertainty relation to write $\mathcal{W}_{1}(|\psi\rangle) \geq V(|\psi\rangle, x)+$ $1 /[(4 V(|\psi\rangle, x)]-1$ for any quadrature $x$, with equality if and only if $|\psi\rangle$ is Gaussian. The minimum of the right-hand side is zero attained at $V(|\psi\rangle, x)=1 / 2$.

For an $n$-mode pure state, we can extend both quantities by considering the covariance matrix defined for an arbitrary mixed state $\rho$ as

$$
V_{s t}(\rho):=\operatorname{Tr}\left[\frac{1}{2}\left\{q_{s}, q_{t}\right\} \rho\right]-\operatorname{Tr}\left[q_{s} \rho\right] \operatorname{Tr}\left[q_{t} \rho\right] .
$$

In this description, PL unitaries correspond to the set of $2 n \times 2 n$ orthogonal symplectic matrices $R \in K(n):=$ $O(2 n) \cap S p(2 n) \cong U(n)$, namely, $R^{T} R=R R^{T}=I$ and $R^{T} \Omega R=\Omega$ [53]. For any phase-space direction $\boldsymbol{r}$, we have $V(\rho, \boldsymbol{r} \cdot \boldsymbol{q})=\boldsymbol{r}^{T} V(\rho) \boldsymbol{r}$.

It follows that $\mathcal{V}_{1}$ is identified with the maximal eigenvalue of $V-I / 2$. To capture multimode structure, we consider the maximal variance over all linear subspaces in phase space of dimension $k \leq 2 n$ :

$$
\mathcal{V}_{k}(|\psi\rangle):=\max _{T: \operatorname{dim} T=k} \operatorname{Tr}_{T}[V(|\psi\rangle)-I / 2],
$$

where $\operatorname{Tr}_{T}$ denotes a trace of the matrix restricted to subspace $T . \mathcal{V}_{k}$ is manifestly invariant under free unitaries, and clearly $\mathcal{V}_{1} \leq \mathcal{V}_{2} \leq \ldots \leq \mathcal{V}_{2 n}$; all $\mathcal{V}_{k}$ vanish exactly on classical states. $\mathcal{V}_{1}$ picks out the direction with largest variance, while $\mathcal{V}_{2 n}$ is the total variance over the entire phase space and coincides with the macroscopicity measure of Ref. [78]. $\mathcal{V}_{k}$ can be calculated as the sum of the $k$ largest eigenvalues $v_{1} \geq \ldots \geq v_{2 n}$ of $V-I / 2: \mathcal{V}_{k}=\sum_{i=1}^{k} v_{i}$ [79].

For $\mathcal{W}_{k}$, we use the concept of a symplectic subspace [80], essentially the $2 k$-dimensional subspace of the full phase space $\mathbb{R}^{2 n}$ corresponding to a choice of $k \leq n$ modes. Every symplectic subspace can be obtained by applying a rotation $R \in K(n)$ to the subspace spanned by the canonical planes for $\left(x_{1}, p_{1}\right), \ldots,\left(x_{k}, p_{k}\right)$. We denote the number of modes in a symplectic subspace $\mathcal{S}$ by $k=|\mathcal{S}|=\operatorname{dim} \mathcal{S} / 2$. Then,

$$
\mathcal{W}_{k}(|\psi\rangle):=\max _{\mathcal{S}:|\mathcal{S}|=k} \operatorname{Tr}_{\mathcal{S}}[V(|\psi\rangle)-I / 2]
$$

$\mathcal{W}_{k}$ is again invariant under free unitaries, we have $\mathcal{W}_{1} \leq \ldots \leq \mathcal{W}_{n}$, and each $\mathcal{W}_{k}$ vanishes exactly on classical states. Also note that $\mathcal{W}_{k} \leq \mathcal{V}_{2 k}$ and $\mathcal{W}_{n}=\mathcal{V}_{2 n}$. As shown 
in Appendix D, we have $\mathcal{W}_{k}=\sum_{i=1}^{k} w_{i}$, where $w_{1} \geq \ldots w_{n}$ are the doubly degenerate eigenvalues of the matrix

$$
W(|\psi\rangle):=\frac{1}{2}\left[V(|\psi\rangle)+\Omega V(|\psi\rangle) \Omega^{T}-I\right] .
$$

The covariance matrix is additive under tensor products $V(\rho \otimes \sigma)=V(\rho) \oplus V(\sigma)$, so combining the systems amounts to appending one list of $v_{i}$ to the other and reordering. We prove in Appendix $\mathrm{H}$ that the phasespace variances $\mathcal{V}_{k}, \mathcal{W}_{k}$ are monotones for pure state transformations.

Theorem 2. (Monotonicity of phase-space variances for pure states.)

(a) Let $|\psi\rangle \mapsto|\phi\rangle$ with probability $p$ under $\mathcal{P}_{1}$, then

$$
p \mathcal{V}_{k}(|\phi\rangle) \leq \mathcal{V}_{k}(|\psi\rangle), \quad \forall k \leq 2 n,
$$

where $n$ is the number of output modes. [If $2 k$ is larger than the number of input modes, then we take $\mathcal{V}_{k}(|\psi\rangle)=\mathcal{V}_{2 n}(|\psi\rangle)$.]

(b) Let $|\psi\rangle \mapsto\left|\phi_{m}\right\rangle$ with probabilities $p_{m}$ under $\mathcal{P}_{\mathbb{N}}$, then

$$
\sum_{m} p_{m} \mathcal{V}_{2 n}\left(\left|\phi_{m}\right\rangle\right) \leq \mathcal{V}_{2 n}(|\psi\rangle)
$$

The same inequalities hold for $\mathcal{W}_{k}, k \leq n$.

Note that the total variance $\mathcal{V}_{2 n}$ is shown to be a stronger monotone than the other $\mathcal{V}_{k}$. $\mathcal{V}_{2 n}$ is a full ensemble monotone for arbitrary-length adaptive protocols, while feed forward presents an obstacle to the proof of monotonicity for the remaining quantities. An upper bound on the probability $p$ of a transformation with $\mathcal{P}_{1}$ follows from Eq. (10):

$$
p \leq \min _{k=1, \ldots, 2 n} \frac{\sum_{i=1}^{k} v_{i}(|\psi\rangle)}{\sum_{i=1}^{k} v_{i}(|\phi\rangle)},
$$

analogous to the condition in entanglement theory derived by Vidal [81]. Indeed, Eq. (10) can be recast as a weak majorization relation: $p \boldsymbol{v}(|\phi\rangle) \prec_{w} \boldsymbol{v}(|\psi\rangle)$ (see Fig. 2). A bound of the same form holds with $v_{i}$ replaced by $w_{i}$.

To extend the measures to mixed states, we use a convex roof construction. A state $\rho$ can generally be expressed in many different ways as a classical mixture of pure states: $\rho=\sum_{\mu} p_{\mu}\left|\psi_{\mu}\right\rangle\left\langle\psi_{\mu}\right|, p_{\mu} \geq 0, \sum_{\mu} p_{\mu}=1$. (We use sum notation for convenience but must bear in mind that a continuous integral may be necessary in general.) The convex roof of $\mathcal{V}_{k}$ is the minimal average value of its pure state components optimized over all possible decompositions of $\rho$ :

$$
\hat{\mathcal{V}}_{k}(\rho):=\inf _{\left\{p_{\mu},\left|\psi_{\mu}\right\rangle\right\}} \sum_{\mu} p_{\mu} \mathcal{V}_{k}\left(\left|\psi_{\mu}\right\rangle\right) .
$$

(We write inf rather than min since the noncompactness of the state space makes it unclear whether the infimum is attained.)

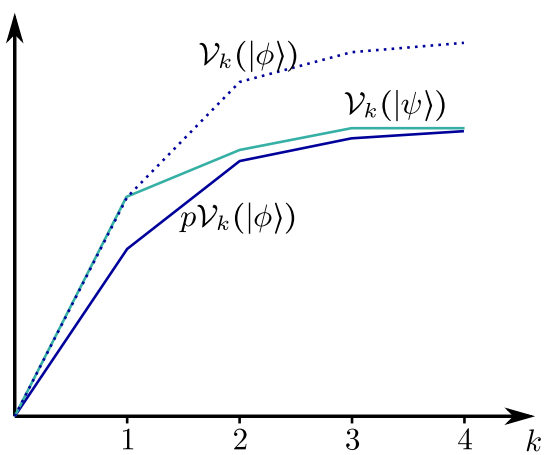

FIG. 2. An illustration of the upper bound (12) on the probability $p$ of reaching a state $|\phi\rangle$ from $|\psi\rangle$ under $\mathcal{P}_{1}$. The curve of $\mathcal{V}_{k}(|\phi\rangle)$ may lie above that of $\mathcal{V}_{k}(|\psi\rangle)$, but it must lie below it when rescaled by $p$.

$\hat{\mathcal{V}}_{k}$ of course satisfies property (iii), being convex by construction. In addition, property (iii) ensures that $\hat{\mathcal{V}}_{k}$ vanishes if $\rho$ is classical. As we show in Appendix E, the converse is also true, but the proof is surprisingly nontrivial due to the infimum in the definition. We show the converse by proving that the trace distance between $\rho$ and $\mathcal{C}_{n}$ is upper bounded by $\hat{\mathcal{V}}_{1}$. Thus, $\hat{\mathcal{V}}_{k}$ is a faithful witness of nonclassicality. The same result holds for $\hat{\mathcal{W}}_{k}$.

Furthermore, as we show in Appendix H, monotonicity carries over from the pure state case thanks to the convex roof construction. Thus, $\hat{\mathcal{V}}_{k}$ and $\hat{\mathcal{W}}_{k}$ are valid measures of nonclassicality for arbitrary states.

One may interpret these measures in terms of quantum fluctuations of quadratures. In the pure state case, they measure the size of quadrature fluctuations above zeropoint motion. With mixed states, one must ensure that the quantified fluctuations are quantum in nature and not due to classical uncertainty - the convexity property (iii) ensures that this is the case.

\section{METROLOGY MONOTONES}

Unfortunately, $\hat{\mathcal{V}}_{k}$ and $\hat{\mathcal{V}}_{k}$ are in general hard to calculate, as the lack of a closed form necessitates numerical optimization. Hence, we provide useful lower bounds using the quantum Fisher information. In general, the QFI is defined not for a single state but for a family of states $\rho_{\theta}$ parametrized by $\theta \in \mathbb{R}$ and measures the rate of change of $\rho_{\theta}$ with respect to $\theta[8]$. When the evolution is generated by an observable $H$ via $\rho_{\theta}=e^{-i \theta H} \rho e^{i \theta H}$, the QFI of a state $\rho$ with respect to $H$ may be defined by $F(\rho, H):=$ $-\left.\partial_{\theta}^{2} \operatorname{Fid}\left(\rho, \rho_{\theta}\right)\right|_{\theta=0}$, where $\operatorname{Fid}(\rho, \sigma)=\operatorname{Tr} \sqrt{\sqrt{\rho} \sigma \sqrt{\rho}}$ is the fidelity between two states. (For later convenience, our QFI is a factor of 4 less than the usual definition.)

With multiple observables, a QFI matrix $F$ can be formed. We are interested in the quadratures as generators, and so we take matrix elements $F_{s t}(\rho):=\frac{1}{4} \operatorname{Tr}\left[\frac{1}{2}\left\{L_{s}, L_{t}\right\} \rho\right]$, $s, t=1, \ldots, 2 n$, where $L_{s}$ is the symmetric logarithmic 
derivative defined implicitly by $\frac{1}{2}\left\{L_{s}, \rho\right\}=-i\left[q_{s}, \rho\right]$. A closed expression can be given in terms of the eigenvectors $\left|\psi_{i}\right\rangle$ and eigenvalues $\lambda_{i}$ of $\rho$ :

$$
F_{s t}=\frac{1}{2} \sum_{i, j} \frac{\left(\lambda_{i}-\lambda_{j}\right)^{2}}{\lambda_{i}+\lambda_{j}}\left\langle\psi_{i}\left|q_{s}\right| \psi_{j}\right\rangle\left\langle\psi_{j}\left|q_{t}\right| \psi_{i}\right\rangle
$$

By linearity with respect to the observables, the QFI of any quadrature $\boldsymbol{r} \cdot \boldsymbol{q}$ is obtained with the quadratic form $F(\rho, \boldsymbol{r} \cdot \boldsymbol{q})=\boldsymbol{r}^{T} F(\rho) \boldsymbol{r}$. The connection with the above measures is the equality for pure states $F(|\psi\rangle)=V(|\psi\rangle)$.

For mixed states, the convexity of the QFI [82] again ensures that classical contributions to the variance are not counted. Measures might be constructed analogously to $\mathcal{V}_{k}(|\psi\rangle)$ by taking traces of $F-I / 2$ over linear subspaces. However, the resulting quantities can be negative. To fix this, we take only the positive part by defining

$$
\mathcal{F}_{k}(\rho):=\max _{T: \operatorname{dim}} \operatorname{Tr}_{T=k}[F(\rho)-I / 2]^{+},
$$

where $[M]^{+}=(|M|+M) / 2$ denotes the positive part of matrix $M$. In terms of the eigenvalues $f_{1} \geq f_{2} \ldots \geq f_{2 n} \geq 0$ of $[F-I / 2]^{+}$, we have $\mathcal{F}_{k}=\sum_{i=1}^{k} f_{i}$.

Analogous to $\mathcal{W}_{k}$, we define

$$
\mathcal{G}_{k}(\rho):=\max _{\mathcal{S}:|\mathcal{S}|=k} \operatorname{Tr}_{\mathcal{S}}[F(\rho)-I / 2]^{+},
$$

and $\mathcal{G}_{k}=\sum_{i=1}^{k} g_{i}$, where $g_{1} \geq \ldots \geq g_{n} \geq 0$ are the doubly degenerate eigenvalues of

$$
G(\rho):=\frac{1}{2}\left[F(\rho)+\Omega F(\rho) \Omega^{T}-I\right]^{+} .
$$

These quantities provide useful lower bounds: $\mathcal{F}_{k} \leq \hat{\mathcal{V}}_{k}$, $\mathcal{G}_{k} \leq \hat{\mathcal{W}}_{k}$. This is because the convex roof is the largest convex function reducing to a specified function on pure states [82]. (Note that while the QFI with respect to a single direction is the convex roof of the variance [83], this does not hold for the eigenvalues of the QFI matrix.) It also follows that they vanish for all classical states. The nonclassicality-witnessing property of the QFI has been noted in Ref. [84]. Furthermore, $\mathcal{F}_{k}$ is more directly accessible in experiments, there being a number of practical techniques for measuring and lower bounding the QFI [85-87].

Remarkably, $\mathcal{F}_{k}$ and $\mathcal{G}_{k}$ are valid monotones, and so they put similar constraints on state transformations.

Theorem 3. (Monotonicity of QFI.)

(a) Let $\rho \mapsto \sigma$ with probability $p$ under $\mathcal{P}_{1}$, then

$$
p \mathcal{F}_{k}(\sigma) \leq \mathcal{F}_{k}(\rho), \quad \forall k \leq 2 n .
$$

Equivalently, $p \boldsymbol{f}(\sigma) \prec_{w} \boldsymbol{f}(\rho)$. (b) Let $\rho \mapsto \sigma_{m}$ with probabilities $p_{m}$ under $\mathcal{P}_{\mathbb{N}}$, then

$$
\sum_{m} p_{m} \mathcal{F}_{2 n}\left(\sigma_{m}\right) \leq \mathcal{F}_{2 n}(\rho)
$$

(c) Let $\rho \mapsto \sigma$ under $\mathcal{P}_{0}$, then

$$
f_{i}(\sigma) \leq f_{i}(\rho), \quad \forall i \leq 2 n .
$$

The same inequalities hold for $\mathcal{G}_{k}, g_{k}, k \leq n$.

Given the operational meaning of the QFI, Theorem 3 has immediate consequences for metrology:

A performance advantage in sensing quadrature displacements cannot be gained with passive linear optics, measurements, and classical resources either deterministically or on average in the case of a probabilistic process.

We see in the next section that this these constraints can be further refined in the context of nonclassicality concentration.

Note also that while $\mathcal{F}_{k}$ and $\mathcal{G}_{k}$ are monotones, they are not strictly full measures of nonclassicality. This is because they do not satisfy condition (i) - they can be zero for some nonclassical states. An example is given by the state $\rho=(1-p) \sum_{n=1}^{\infty} p^{n-1}|n\rangle\langle n|$ (a thermal state with the vacuum term removed). We find $\mathcal{F}_{k}(\rho)=0$ when $p>1 / 2$ (see Appendix G), but this state is always nonclassical [88].

Nevertheless, the monotonicity enables us to still treat these quantities as valid and potentially highly useful quantifiers of nonclassicality. An analogous situation occurs in entanglement theory, where local operations and classical communication (LOCC) operations are considered free. Here, negativity $[89,90]$ - a common quantifier of entanglement-is known to be a monotone under LOCC but may vanish for certain entangled states [90].

\section{CONCENTRATION OF NONCLASSICALITY AND ITS IMPLICATIONS FOR METROLOGY}

\section{A. Concentration of nonclassicality}

The monotonicity of $\hat{\mathcal{V}}_{2 n}, \hat{\mathcal{W}}_{n}, \mathcal{F}_{2 n}, \mathcal{G}_{n}$ immediately implies that the total quantum variance cannot increase on average; i.e., the total amount of nonclassicality is not increased by a free operation. However, the monotonicity of $\hat{\mathcal{V}}_{k}, \hat{\mathcal{W}}_{k}, \mathcal{F}_{k}, \mathcal{G}_{k}$ for general $k$ offers a family of more refined constraints.

Specifically, consider the task of nonclassicality concentration, where a process is to output a subset of modes with higher values of nonclassicality than they originally had, while the remaining modes reduce in value. The above constraints specify that such a task can be performed only with a certain maximal probability.

To illustrate this, consider the following scheme for "growing cat states" [91]. Starting with a pair of cat states $\left|\psi_{c}(\alpha)\right\rangle^{\otimes 2}$ with $|\alpha| \gg 1$, we have $\mathcal{W}_{1}=|\alpha|^{2}$, 
$\mathcal{W}_{2}=2|\alpha|^{2}$. Interacting the modes at a 50:50 beam splitter gives $|\sqrt{2} \alpha\rangle|0\rangle+|0\rangle|\sqrt{2} \alpha\rangle+|0\rangle|-\sqrt{2} \alpha\rangle+|-\sqrt{2} \alpha\rangle|0\rangle$. Performing a measurement projecting one mode onto the vacuum, with probability $1 / 2$ the other mode becomes $\left|\psi_{c}(\sqrt{2} \alpha)\right\rangle$, i.e., $\mathcal{W}_{1}=2|\alpha|^{2}$ has doubled. While this was observed to be the optimal probability for outputting a double-sized cat state for the scheme in Ref. [91], Theorem 2(a) proves that in fact no other protocol can perform better.

Theorem 3(c) gives additional, stronger constraints for $\mathcal{P}_{0}$ transformations. They highlight that there is a strict hierarchy in terms of the power of the different sets of free operations: Concentration of nonclassicality is impossible without measurement. (Theorem 4 below extends this statement to the full $\mathcal{P}_{\mathbb{N}}$ set of operations for the case of Gaussian states.)

\section{B. Metrological implications}

We begin with the operational interpretation of the relevant quantities. First, $\mathcal{F}_{1}$ represents the optimal performance of a state for sensing a displacement in any single direction (i.e., optimized over all directions in phase space). More generally, $\mathcal{F}_{k}$ indicates the optimal performance for simultaneous sensing of $k$ orthogonal directions, where an equal weighting is given to each direction. Note that the trace of the QFI is a commonly used figure of merit in multiparameter metrology [92]. A similar interpretation holds for $\mathcal{G}_{k}$, where the $2 k$ directions are now also required to form a symplectic basis (i.e., $k$ orthogonal conjugate pairs of directions). Theorem 3(a) may thus be rephrased as the following statement:

With only passive linear optics, measurements, and classical resources, the conversion of a state which is useful for multiparameter metrology into one which is more useful for estimating fewer parameters, necessarily has a limited probability of success.

Moreover, the eigenvalues $f_{i}$ of the QFI matrix may be understood as the ability to estimate displacements along the $i$ th "best" direction in phase space for a given state; i.e., $f_{1}$ is the most sensitive direction, $f_{2}$ is the second most sensitive, and so on. Let us refer to the corresponding directions in phase space as principal directions (note that they form a set of orthogonal axes). Theorem 3(c) gives a stronger constraint for $\mathcal{P}_{0}$ transformations than the first parts of the theorem:

With only passive linear optics and classical resources, the sensitivity to displacements along any principal direction cannot increase.

\section{GAUSSIAN STATES}

Gaussian states $[53,93,94]$ are fully determined by their first and second quadrature moments. Since displacements are free operations, here we lose no generality by neglecting the first moments $\operatorname{Tr}\left(\rho q_{s}\right)$ and characterizing states only by their covariance matrices. A simple condition for nonclassicality of Gaussian states is known: $\rho$ is nonclassical if and only if the smallest eigenvalue of $V(\rho)$ satisfies $v_{\min }<1 / 2$ [95]. This "squeezing criterion" simply tests whether there exists a quadrature that exhibits less noise than the vacuum.

It may be shown (see Appendix I) that for Gaussian states, $F=\frac{1}{4} \Omega V^{-1} \Omega^{T}$. Hence, all of the above constraints on state transformations can be expressed in terms of eigenvalues of $V$. Moreover, the squeezing criterion implies that $f_{1}=0$ if and only if $\rho$ is classical; unlike the general case, $f_{i}, g_{i}$ and $\mathcal{F}_{k}, \mathcal{G}_{k}$ are valid measures of nonclassicality for Gaussian states.

We look at the case where input and output both have $n$ modes. Lemma 1 then says that the ancilla used in a $\mathcal{P}_{1}$ instrument can be assumed to have $n$ modes (the same conclusion was reached via a different argument in Ref. [51]). While $\mathcal{P}_{0}$ necessarily preserves Gaussianity, measurements and feed forward must be constrained. A Gaussian measurement is described by POVM elements of the form $E(\boldsymbol{\alpha})=\pi^{-n} D(\boldsymbol{\alpha}) \Lambda D(\boldsymbol{\alpha})^{\dagger}$, where $\Lambda$ is a Gaussian state [93]. We constrain the conditional feed-forward operations to be displacements with linear gain (such as those in Ref. [68]). We refer to the resulting Gaussian set of free operations as $\mathcal{G} \mathcal{P}_{\mathbb{N}}$. The resulting resource theory of squeezing is a subresource theory of nonclassicality.

Theorem 4. (No concentration of nonclassicality for Gaussian states.) Let Gaussian $\rho \mapsto \sigma$ under $\mathcal{G P}_{\mathbb{N}}$ with nonzero probability, then

$$
f_{i}(\sigma) \leq f_{i}(\rho), \quad \forall i \leq 2 n,
$$

and similarly for $g_{i}, i \leq n$.

This result shows that concentration of nonclassicality is impossible in the Gaussian case (see Appendix H). It echoes similar no-go results about distillation of resources in Gaussian settings [96-99].

We now determine some necessary and sufficient conditions for Gaussian state transformations. The single-mode case is particularly simple: Since $K(1) \cong U(1) \cong S O(2), V$ can be diagonalized with a PL unitary $R \in K(1)$. Hence, we need to only keep track of its eigenvalues $v_{+} \geq v_{-}$. We define three measures of nonclassicality:

$$
\begin{aligned}
& \mathcal{N}_{1}:=\max \left\{1-2 v_{-}, 0\right\} \\
& \mathcal{N}_{2}:=\frac{\mathcal{N}_{1}}{2 v_{+}-1} \\
& \mathcal{N}_{3}:=v_{+} \mathcal{N}_{2}=\frac{\mathcal{N}_{1}}{2-\frac{1}{v_{+}}}
\end{aligned}
$$

Theorem 5. (Single-mode Gaussian conversion.)

(a) Gaussian $\rho \mapsto \sigma$ under $\mathcal{P}_{0}$ if and only if $\mathcal{N}_{1}(\sigma) \leq$ $\mathcal{N}_{1}(\rho)$ and $\mathcal{N}_{2}(\sigma) \leq \mathcal{N}_{2}(\rho)$. 


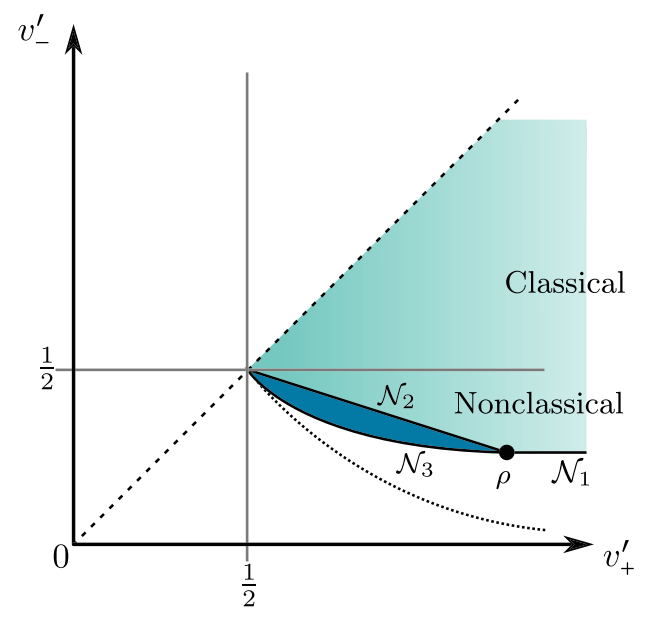

FIG. 3. The region of achievable states from a single-mode Gaussian state $\rho$ under free operations. The light shaded region is accessible under $\mathcal{P}_{0}$, while $\mathcal{G} \mathcal{P}_{\mathbb{N}}$ transformations also access the dark shaded region. The constraints provided by the monotones $\mathcal{N}_{i}$ are shown. The dotted curve delimits the physical states satisfying the uncertainty relation $v_{+} v_{-} \geq 1 / 4$.

(b) Gaussian $\rho \mapsto \sigma$ under $\mathcal{G} \mathcal{P}_{\mathbb{N}}$ with nonzero probability if and only if $\mathcal{N}_{1}(\sigma) \leq \mathcal{N}_{1}(\rho)$ and $\mathcal{N}_{3}(\sigma) \leq \mathcal{N}_{3}(\rho)$.

(See Appendix $\mathrm{J}$ for the proof.) $\mathcal{N}_{1}$ is simply a measure of squeezing, having minimum value zero for classical states and maximum 1 for infinitely squeezed states. $\mathcal{N}_{2}$, $\mathcal{N}_{3}$ place limits on the amount of noise that can be removed from the larger-variance quadrature. With $\mathcal{P}_{0}$, noise is best removed by mixing with a coherent state at a beam splitter. With $\mathcal{G} \mathcal{P}_{\mathbb{N}}$, one can perform a homodyne measurement on the ancilla, thereby reducing the uncertainty in one direction. In both cases, a reduction in noise comes with an associated loss of squeezing. The region of accessible states is illustrated in Fig. 3.

Under $\mathcal{P}_{0}$, a single-mode pure state $|\psi\rangle$ cannot be sent to any pure state other than itself or a coherent state. Since the uncertainty relation is saturated, i.e., $v_{+} v_{-}=1 / 4$, we have $\mathcal{N}_{3}(|\psi\rangle)=1 / 2$, and $\mathcal{N}_{1}$ becomes the only nontrivial monotone under $\mathcal{G} \mathcal{P}_{\mathbb{N}}$. Thus, $|\psi\rangle$ can be transformed under $\mathcal{G} \mathcal{P}_{\mathbb{N}}$ into any less-squeezed state $\rho$, i.e., with a lower value of $\mathcal{N}_{1}$.

For $n$-mode pure states, we again have the simplification that $V$ is diagonalized by $R \in K(n)$ :

$$
R^{T} V R=\bigoplus_{i=1}^{n} \frac{1}{2}\left(\begin{array}{cc}
s_{i} & 0 \\
0 & s_{i}^{-1}
\end{array}\right)
$$

where the $s_{1} \geq \ldots \geq s_{n} \geq 1$ are squeezing parameters [100]. Physically, $|\psi\rangle$ can be disentangled into $n$-independent squeezed states $\left|s_{1}\right\rangle\left|s_{2}\right\rangle, \ldots,\left|s_{n}\right\rangle$ by a PL unitary. Thus, full conditions for transformations between Gaussian pure states can be given.
Theorem 6. (n-mode pure Gaussian conversion.) A Gaussian pure state $|\psi\rangle \mapsto|\phi\rangle$ under $\mathcal{G P}_{\mathbb{N}}$ if and only if $s_{i}(|\phi\rangle) \leq s_{i}(|\psi\rangle) \forall i$.

The necessity of the inequalities follows from Theorem 4 after noting that $f_{i}=s_{i} / 2$ for $i=1, \ldots, n$. Conversely, given the inequalities, the above observation on singlemode pure state transformations shows that an operation effecting the transformation exists: diagonalize $|\psi\rangle$ with a PL unitary, operate on each squeezed mode individually, then apply a suitable PL unitary to get $|\phi\rangle$.

\section{CONCLUSIONS}

Recent advances in quantum resource theories have led to sophisticated tools for identifying and quantifying nonclassicality-features of quantum information that distinguish it from classical counterparts. This article adapts these tools to identify an operational resource theory of nonclassicality in the continuous-variable regime. Our approach is to take those classicality-preserving operations which are considered comparatively easy to engineer in experimental conditions, namely, passive linear unitaries and measurements with feed forward. Quadrature variances and quantum Fisher information emerge naturally within this framework as quantifiers of nonclassicality. These then provide strong and general bounds regarding concentration of quantum resources. In the Gaussian regime, our framework reveals a hierarchy of nonclassicality beyond squeezing. Meanwhile, no-go theorems for squeezing concentration fall out as corollaries, providing a new perspective on recent results in Gaussian resources theories [99].

Another noteworthy point is the significance of quantum Fisher information. Commonly used as a measure of performance in parameter estimation, its emergence here indicates an operational interpretation of continuous-variable nonclassicality as a resource for metrological applications. While not every nonclassical state is useful for metrology, we show that ranking one state as more nonclassical than another implies a higher precision in sensing quadrature displacements. Meanwhile, these practical consequences directly lead to experimental means of verifying nonclassicality within a given system, without resorting to full tomography. Quantum Fisher information has also recently been adopted to quantify the macroscopicity of quantum systems $[74,86,101]$. This connection hints that in capturing an operational form of nonclassicality for continuous-variable systems, we may naturally recover innate notions of macroscopic quantum effects.

We expect our results to lay the foundations for a systematic understanding of nonclassicality in the continuous-variable regime. Certainly many exciting questions remain. One direction is to extend our result to independent and identically distributed scenarios, where one seeks to convert $N$ copies of a quantum state to another without additional sources of nonclassicality-in the limit of large $N$. Could we use these ideas to build a hierarchy of 
nonclassicality for general, possibly non-Gaussian, continuous-variable states? Progress in this direction will ultimately help us fully characterize the distinguishing features of nonclassical light.

\section{ACKNOWLEDGMENTS}

We would like to thank Hyukjoon Kwon, Florian Fröwis, Howard Wiseman, Syed Assad, Mark Bradshaw, Ping Koy Lam, Tim Ralph, and Farid Shahandeh for helpful discussions. This work is supported by UK EPSRC (Grant No. EP/K034480/1 and Doctoral Prize), the KIST Institutional Program No. 2E26680-18-P025, the National Research Foundation of Singapore Fellowship No. NRF-NRFF2016-02, the National Research Foundation and L'Agence Nationale de la Recherche joint Project No. NRF2017-NRF-ANR004 VanQuTe, and the Singapore Ministry of Education Tier 1 RG190/17. M. S. K. acknowledges the Samsung GRO project and the Royal Society for their financial support.

Note added.-Recently, we were made aware of a related paper by Kwon et al. [102], which appears concurrently.

\section{APPENDIX A: QUANTUM-INSTRUMENT DEFINITION OF FREE OPERATIONS}

Here we give a formal quantum-instrument definition of the free operations. We employ the concepts which were introduced in Ref. [103] to study local operations and classical communication. As we mention in the main text, a quantum instrument is a family of completely positive (CP) maps $\mathcal{I}=\left(\mathcal{A}_{m}\right)_{m}$ such that $\sum_{m} \mathcal{A}_{m}$ is trace preserving. Although the inputs and outputs may have different numbers of modes, we assume that each CP map in a given instrument has the same sized inputs and outputs.

We say that $\mathcal{I}^{\prime}=\left(\mathcal{A}_{l}^{\prime}\right)_{l}$ is a coarse graining of $\mathcal{I}=$ $\left(\mathcal{A}_{m}\right)_{m}$ when the index set of $\mathcal{I}$ is partitioned into sets $\Sigma_{l}$ such that $\mathcal{A}_{l}^{\prime}=\sum_{m \in \Sigma_{l}} \mathcal{A}_{m}$.

Any instrument $\mathcal{I} \in \mathcal{P}_{0}$ has a single element: $\mathcal{I}=(\mathcal{A})$. Given a set of input system modes $S$ and ancilla modes $A$, we pass the state $\rho_{S} \otimes \rho_{A}$, where $\rho_{A}$ is classical through a PL unitary $U$ and trace out some set of modes $A^{\prime}$ to give an output $\sigma_{S^{\prime}}$. Hence,

$$
\sigma_{S^{\prime}}=\mathcal{A}\left(\rho_{S}\right)=\operatorname{Tr}_{A^{\prime}}\left[U\left(\rho_{S} \otimes \rho_{A}\right) U^{\dagger}\right] .
$$

For $\mathcal{P}_{1}$, we let an arbitrary POVM $\left\{E^{m}\right\}_{m}, E^{m} \geq 0$, $\sum_{m} E^{m}=I$ act on $A^{\prime}$. Then,

$$
\mathcal{A}_{m}\left(\rho_{A}\right)=\operatorname{Tr}_{A^{\prime}}\left[E_{A^{\prime}}^{m} U\left(\rho_{S} \otimes \rho_{A}\right) U^{\dagger}\right] .
$$

To describe multiple rounds with feed forward, we introduce the following terminology: $\mathcal{I}^{\prime}=\left(\mathcal{A}_{l}^{\prime}\right)_{l}$ is PL linked to $\mathcal{I}=\left(\mathcal{A}_{m}\right)_{m}$ if there exists a collection of $\mathcal{J}_{m}=\left(\mathcal{B}_{l \mid m}\right)_{l} \in$ $\mathcal{P}_{1}$ such that $\mathcal{I}^{\prime}$ is a coarse graining of $\left(\mathcal{B}_{l \mid m} \circ \mathcal{A}_{m}\right)_{m, l}$.
Operationally, $\mathcal{I}$ is performed and the measurement outcome retained; a new $\mathcal{P}_{1}$ operation is then performed conditional on the previous measurement; finally, some forgetting of classical information may happen.

Finally, we then say that $\mathcal{I} \in \mathcal{P}_{r}$ for $r \geq 2$ if $\mathcal{I}$ is PL linked to an element of $\mathcal{P}_{r-1}$. The set of all finite-length protocols is $\mathcal{P}_{\mathbb{N}}=\bigcup_{r=1}^{\infty} \mathcal{P}_{r}$. Note that while coarse graining can be performed after each measurement, it is always possible to assume that this happens only at the end of the protocol. In addition, every protocol can be described as a coarse graining of a fine-grained instrument in which each $\mathcal{A}_{m}$ has a single Kraus operator, $\mathcal{A}_{m}(\rho)=K_{m} \rho K_{m}^{\dagger}$.

\section{APPENDIX B: MAXIMUM REQUIRED ANCILLA SIZE}

We first establish a useful statement about the structure of PL unitaries.

Lemma 2. Let $U$ be a PL unitary taking two sets of input modes $A, B$ of sizes $n_{A}, n_{B}$ and outputting two sets $C$, $D$ of sizes $n_{C}, n_{D}$. There exists a decomposition of $U$ into $b$ beam splitters taking $A_{1} B_{1} \rightarrow C_{1} D_{1}, \ldots, A_{b} B_{b} \rightarrow C_{b} D_{b}$, where $b=\min \left\{n_{A}, n_{B}, n_{C}, n_{D}\right\}$, and initial and final PL unitaries $X_{A}, X_{B}, Y_{C}, Y_{D}$ acting on each group separately. There remaining modes are transferred between groups (see Fig. 4).

Proof.-This is a direct consequence of a matrix decomposition result known as the cosine-sine (CS) decomposition [104]. In its most general form, this states that, given partitions $n=n_{A}+n_{B}=n_{C}+n_{D}$, a matrix $U \in U(n)$ can be written as $U=Y D X$, where

$$
\begin{aligned}
& X=\left(\begin{array}{cc}
X_{A} & \\
& X_{B}
\end{array}\right), \quad Y=\left(\begin{array}{ll}
Y_{C} & \\
& Y_{D}
\end{array}\right), \\
& D=\left(\begin{array}{ccc|ccc}
C & & & S & & \\
& I & & & O_{s}^{T} & \\
& & O_{c} & & & I \\
\hline S & & & -C & & \\
& O_{s} & & & I & \\
& & I & & & O_{c}^{T}
\end{array}\right),
\end{aligned}
$$

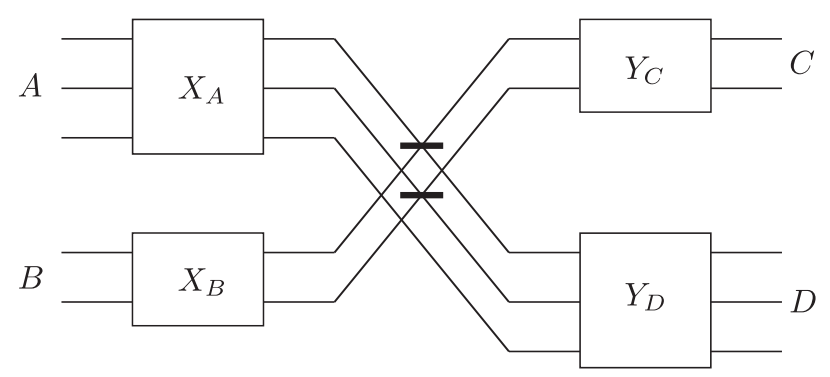

FIG. 4. The decomposition of Lemma 2 for the case $\left(n_{A}, n_{B}, n_{C}, n_{D}\right)=(3,2,2,3)$. 


$$
\begin{array}{cc}
C=\operatorname{diag}\left(c_{1}, \ldots, c_{b}\right), & 1 \geq c_{1} \geq \ldots \geq c_{b} \geq 0, \\
S=\operatorname{diag}\left(s_{1}, \ldots, s_{b}\right), & 0 \leq s_{1} \leq \ldots \leq s_{b} \leq 1, \\
C^{2}+S^{2}=I .
\end{array}
$$

Here, $X_{A}, X_{B}, Y_{C}, Y_{D}$ are unitaries of respective dimensions $n_{A}, n_{B}, n_{C}, n_{D}$, and $D$ is shown partitioned into blocks of $n_{C}, n_{D}$ rows and $n_{A}, n_{B}$ columns. The identity matrices $I$ are not all the same size, and $O_{c}, O_{s}$ are rectangular matrices of zeroes whose sizes depend on the partition.

$X$ and $Y$ correspond to the initial and final "local" unitaries. To interpret the form of $D$, first note that the subblock containing the $C$ and $S$ matrices corresponds to beam splitters of reflectivities $c_{i}^{2}$ between the first $b$ modes of $A$ and $B$. The remaining sub-blocks correspond to direct transferral of modes from $A \rightarrow C$ and $B \rightarrow D$, as well as swapping $A \rightarrow D$ and $B \rightarrow C$.

Now consider a $\mathcal{P}_{1}$ operation taking input system $S$ and ancilla $A$ to output system $S^{\prime}$ and ancilla $A^{\prime}$, where a final measurement is performed on $A^{\prime}$. We first show that if $n_{A}>n_{S^{\prime}}$, then there are ancilla modes which do not interact with the system. Later, we show that these modes are unnecessary. (In the following, we can ignore the local PL unitaries $X_{S}, X_{A}, Y_{S^{\prime}}, Y_{A^{\prime}}$ acting on each group without loss of generality.) Suppose first that $n_{S} \geq n_{S^{\prime}}$. Then, by applying Lemma 2 to the input groupings $S, A$ and output groupings $S^{\prime}, A^{\prime}$, at most $n_{S^{\prime}}$ beam splitters are needed. So only $n_{S^{\prime}}$ of the modes in $A$ are required for the beam splitters, while the remainder go directly to $A^{\prime}$. If instead $n_{S} \leq n_{S^{\prime}}$, then only $n_{S}$ beam splitters are needed. $n_{S}$ of the outputs from the beam splitters go to $S^{\prime}$; to make up the remainder, $n_{S^{\prime}}-n_{S}$ modes from $A$ must be transferred to $S^{\prime}$. So in total, no more than $\left(n_{S^{\prime}}-n_{S}\right)+n_{S}=n_{S^{\prime}}$ modes are needed for $A$, apart from those going directly into $A^{\prime}$.

Suppose that $n_{A}>n_{S^{\prime}}$. By the above argument, we can divide $A$ into two sets of modes $B, C$ and similarly $A^{\prime}$ into $B^{\prime}, C^{\prime}$, such that $B$ interacts with $S$ via beam splitters with possible transferral of modes, $C$ maps directly onto $C^{\prime}$, and $B^{\prime}$ contains all other measured modes (see Fig. 5). Since the ancilla is initially classical, we can write

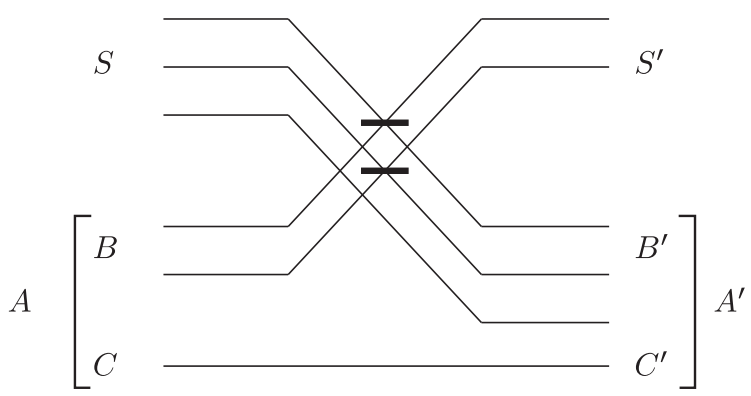

FIG. 5. An example of unnecessary ancilla modes $\left(C, C^{\prime}\right)$ for a $\mathcal{P}_{1}$ operation.
$\rho_{B C}=\int \mathrm{d}^{2 n_{C^{\prime}}} \gamma P(\gamma) \rho_{B \mid \gamma} \otimes|\gamma\rangle\left\langle\left.\gamma\right|_{C}, \quad P(\gamma) \geq 0\right.$,

where $|\gamma\rangle$ is a coherent state on $C$. Let $\sigma_{S^{\prime} B^{\prime} \mid \gamma}$ be the result of applying the PL unitary to $\rho_{S} \otimes \rho_{B \mid \gamma}$ and transferring any necessary modes from $S$ to $B^{\prime}$ and $B$ to $S^{\prime}$. This transformation describes the dynamics of all modes apart from $C$ going to $C^{\prime}$. Thus, with a POVM $\left\{E_{B^{\prime} C^{\prime}}^{m}\right\}$, the output state is

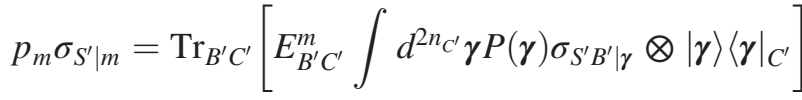

$$
\begin{aligned}
& =\operatorname{Tr}_{B^{\prime}}\left[\int d^{2 n_{C^{\prime}}} \gamma P(\gamma) F_{B^{\prime} \mid \gamma}^{m} \sigma_{S^{\prime} B^{\prime} \mid \gamma}\right],
\end{aligned}
$$

where $F_{B^{\prime} \mid \gamma}^{m}:=\left\langle\left.\gamma\right|_{C^{\prime}} E_{B^{\prime} C^{\prime}}^{m} \mid \gamma\right\rangle_{C^{\prime}}$ defines a new POVM for each $\gamma$. Thus, we see that the $C$ modes are unnecessary and just result in a classical mixture of different $\mathcal{P}_{1}$ protocols.

\section{APPENDIX C: KRAUS OPERATORS FOR $\mathcal{P}_{\mathbb{N}}$}

First note that every quantum operation is uniquely determined by its action on coherent states due to the $P$ representation and the linearity of operations. So, we need to consider only fine-grained elements of $\mathcal{P}_{\mathbb{N}}$ in which all ancilla states are initially pure (i.e., coherent states) and all measurements are rank-1 POVMs. We look at a single such element of $\mathcal{P}_{1}$ with input $|\boldsymbol{\alpha}\rangle_{S}$ and initial ancilla state $|\boldsymbol{\beta}\rangle_{A}$. Before the measurement, we have the state $\left|\boldsymbol{\alpha}^{\prime}\right\rangle_{S^{\prime}}\left|\boldsymbol{\beta}^{\prime}\right\rangle_{A^{\prime}}$, where

$$
\left(\begin{array}{l}
\boldsymbol{\alpha}^{\prime} \\
\boldsymbol{\beta}^{\prime}
\end{array}\right)=\left(\begin{array}{ll}
U_{11} & U_{12} \\
U_{21} & U_{22}
\end{array}\right)\left(\begin{array}{l}
\boldsymbol{\alpha} \\
\boldsymbol{\beta}
\end{array}\right)
$$

where $U_{11}$ is $n_{S^{\prime}} \times n_{S}, U_{12}$ is $n_{S^{\prime}} \times n_{A}$, and so on. It is immediately clear from the decomposition in Lemma 2 that $U_{11}$ has singular values of modulus $\leq 1$. Hence, $\boldsymbol{\alpha}^{\prime}=U_{11} \boldsymbol{\alpha}+U_{12} \boldsymbol{\beta}$, where $U_{11}$ is a contraction, and the displacement by $U_{12} \boldsymbol{\beta}$ is independent of the input $\boldsymbol{\alpha}$.

Since $\left|\boldsymbol{\alpha}^{\prime}\right\rangle_{S^{\prime}}\left|\boldsymbol{\beta}^{\prime}\right\rangle_{A^{\prime}}$ is uncorrelated, any measurement on $A^{\prime}$ has no backaction on $S^{\prime}$. Suppose a rank-1 POVM has outcome $m$ projecting $A^{\prime}$ onto $\left|\psi_{m}\right\rangle$, then the Kraus operator determining the mapping from input to output is

$$
K_{m}|\boldsymbol{\alpha}\rangle_{S}=c_{m}(\boldsymbol{\alpha})\left|U_{11} \boldsymbol{\alpha}+U_{12} \boldsymbol{\beta}\right\rangle_{S^{\prime}},
$$

where $c_{m}(\boldsymbol{\alpha})=\left\langle\psi_{m} \mid U_{21} \boldsymbol{\alpha}+U_{22} \boldsymbol{\beta}\right\rangle$.

Finally, it is clear that the Kraus operator form remains the same under multiple rounds with feed forward, where the contractions and displacements applied may depend on previous measurement outcomes, hence, the stated form of general $K_{m}$ for $\mathcal{P}_{\mathbb{N}}$. 


\section{APPENDIX D: EIGENVALUE EXPRESSION FOR $\mathcal{W}_{k}$}

$W$ has a particular structure in terms of $2 \times 2$ blocks,

$$
W=\left(\begin{array}{ccc}
W^{11} & W^{12} & \ldots \\
W^{21} & W^{22} & \ldots \\
\vdots & \vdots & \ddots
\end{array}\right), \quad W^{i j}=\left(\begin{array}{cc}
W_{R}^{i j} & -W_{I}^{i j} \\
W_{I}^{i j} & W_{R}^{i j}
\end{array}\right),
$$

where each diagonal block has $W_{I}^{i i}=0$. This structure lets us use an isomorphism [105] onto a complex vector space of half the dimension: We form $\tilde{W} \in \mathbb{C}^{n \times n}$ with elements $\tilde{W}_{i j}:=W_{R}^{i j}+i W_{I}^{i j}$, and similarly, a vector $\boldsymbol{r}=$ $\left(r_{1, x}, r_{1, p}, r_{2, x}, r_{2, p}, \ldots\right) \in \mathbb{R}^{2 n}$ is mapped to $\tilde{\boldsymbol{r}}=\left(r_{1, x}+\right.$ $\left.i r_{1, p}, r_{2, x}+i r_{2, p}, \ldots\right) \in \mathbb{C}^{n}$. Then, $\tilde{\boldsymbol{r}}^{\dagger} \tilde{W} \tilde{\boldsymbol{r}}=\boldsymbol{r}^{T} W \boldsymbol{r}$; in addition, an orthogonal basis in $\mathbb{C}^{n}$ corresponds to a symplectic basis in $\mathbb{R}^{2 n}$. Therefore,

$$
\mathcal{W}_{k}=\max _{T: \operatorname{dim} T=k} \operatorname{Tr}_{T} \tilde{W},
$$

where the maximization is over all subspaces $T$ of complex dimension $k$.

Note that $\tilde{W}$ is Hermitian and positive semidefinite; the Courant-Fischer theorem [79] states that its eigenvalues $w_{1} \geq w_{2} \geq, \ldots, w_{n} \geq 0$ can be obtained by

$$
\begin{aligned}
w_{i} & =\max _{T: \operatorname{dim}} \min _{T=i} \boldsymbol{c}_{\boldsymbol{c} \in T:|c|=1}^{\dagger} \tilde{W} \boldsymbol{c} \\
& =\max _{\mathcal{S}:|\mathcal{S}|=i} \min _{\boldsymbol{r} \in \mathcal{S}:|\boldsymbol{r}|=1} \boldsymbol{r}^{T} W \boldsymbol{r},
\end{aligned}
$$

and moreover, $\mathcal{W}_{k}=\sum_{i=1}^{k} w_{i}$. The fact that $w_{i}$ are the doubly degenerate eigenvalues of $W$ is evident from inverting the isomorphism to map from the diagonal form of $\tilde{W}$ back to the real $2 n$-dimensional matrix $\operatorname{diag}\left(w_{1}, w_{1}, w_{2}, w_{2}, \ldots\right)$.

\section{APPENDIX E: VANISHING OF CONVEX ROOF}

Here we show that $\hat{\mathcal{V}}_{k}(\rho)=0 \Rightarrow \rho \in \mathcal{C}_{n}$. This is not straightforward because of the infimum in the definition. We start with an inequality showing that small $\hat{\mathcal{V}}_{k}(\rho)$ implies $\rho$ is close to $\mathcal{C}_{n}$. We use the nonclassical distance as defined in Ref. [19]:

$$
\delta(\rho):=\inf _{\sigma \in \mathcal{C}_{n}} D_{\operatorname{Tr}}(\rho, \sigma),
$$

where $D_{\operatorname{Tr}}(\rho, \sigma):=\frac{1}{2} \operatorname{Tr}|\rho-\sigma|$ is the trace distance [106].

Lemma 3. For an $n$-mode pure state $|\psi\rangle$, there exists a classical pure state $|\phi\rangle$ such that

$$
D_{\operatorname{Tr}}(|\psi\rangle,|\phi\rangle)^{2} \leq n \mathcal{V}_{1}(|\psi\rangle) .
$$

Proof.-We initially assume for simplicity that $|\psi\rangle$ has vanishing first moments. Choose a set of modes with quadratures $x_{i}, p_{i}$ such that $x_{1}$ is the optimal quadrature with $\mathcal{V}_{1}(|\psi\rangle)=V\left(|\psi\rangle, x_{1}\right)-1 / 2$. Defining number operators $N_{i}=\left(x_{i}^{2}+p_{i}^{2}-1\right) / 2$ and $N=\sum_{i=1}^{n} N_{i}$, we have

$$
\begin{aligned}
\langle N\rangle & =\frac{1}{2} \sum_{i=1}^{n}\left[V\left(|\psi\rangle, x_{i}\right)+V\left(|\psi\rangle, p_{i}\right)-1\right] \\
& \leq n\left(V\left(|\psi\rangle, x_{1}\right)-\frac{1}{2}\right) \\
& =n \mathcal{V}_{1}(|\psi\rangle) .
\end{aligned}
$$

The overlap between $|\psi\rangle$ and the $n$-mode vacuum state is $|\langle 0 \mid \psi\rangle|^{2}=P(N=0)$. By writing $\langle N\rangle=\sum_{k=0}^{\infty} k P(N=k) \geq$ $\sum_{k=1}^{\infty} P(N=k)$, we obtain

$$
|\langle 0 \mid \psi\rangle|^{2}=1-P(N \geq 1) \geq 1-\langle N\rangle \geq 1-n \mathcal{V}_{1}(|\psi\rangle) .
$$

For pure states, the trace distance simplifies to $D_{\operatorname{Tr}}(|\psi\rangle$, $|0\rangle)=\sqrt{1-|\langle 0 \mid \psi\rangle|^{2}} \quad[106]$, hence, $\quad D_{\operatorname{Tr}}(|\psi\rangle,|0\rangle)^{2} \leq$ $n \mathcal{V}_{1}(|\psi\rangle)$. Finally, in general we take $|\phi\rangle$ to be the coherent state with the same first moments as $|\psi\rangle$.

If $\hat{\mathcal{V}}_{1}(\rho)=0$, then there exists a sequence of pure state decompositions such that $\rho=\sum_{\mu} p_{\alpha, \mu}\left|\psi_{\alpha, \mu}\right\rangle\left\langle\psi_{\alpha, \mu}\right|$ for each $\alpha=0,1,2, \ldots$, and $\lim _{\alpha \rightarrow \infty} \sum_{\mu} p_{\alpha, \mu} \mathcal{V}_{1}\left(\left|\psi_{\alpha, \mu}\right\rangle\right)=0$. So, for any $\epsilon>0$, there exists $\alpha^{*}$ such that

$$
\sum_{\mu} p_{\alpha, \mu} \mathcal{V}_{1}\left(\left|\psi_{\alpha}\right\rangle\right) \leq \epsilon, \quad \forall \alpha \geq \alpha^{*}
$$

Applying Lemma 3, we find a sequence of classical $\left|\phi_{\alpha, \mu}\right\rangle$ such that

$$
\sum_{\mu} p_{\alpha, \mu} D_{\operatorname{Tr}}\left(\left|\psi_{\alpha, \mu}\right\rangle,\left|\phi_{\alpha, \mu}\right\rangle\right)^{2} \leq n \epsilon, \quad \forall \alpha \geq \alpha^{*} .
$$

The Cauchy-Schwarz inequality gives

$$
\begin{aligned}
& \left(\sum_{\mu} p_{\alpha, \mu} D_{\operatorname{Tr}}\left(\left|\psi_{\alpha, \mu}\right\rangle,\left|\phi_{\alpha, \mu}\right\rangle\right)\right)^{2} \\
& =\left(\sum_{\mu} \sqrt{p_{\alpha, \mu}} \times \sqrt{p_{\alpha, \mu}} D_{\operatorname{Tr}}\left(\left|\psi_{\alpha, \mu}\right\rangle,\left|\phi_{\alpha, \mu}\right\rangle\right)\right)^{2} \\
& \leq\left(\sum_{\mu} p_{\alpha, \mu}\right)\left(\sum_{\mu} p_{\alpha, \mu} D_{\operatorname{Tr}}\left(\left|\psi_{\alpha, \mu}\right\rangle,\left|\phi_{\alpha, \mu}\right\rangle\right)^{2}\right) \\
& \quad=\sum_{\mu} p_{\alpha, \mu} D_{\operatorname{Tr}}\left(\left|\psi_{\alpha, \mu}\right\rangle,\left|\phi_{\alpha, \mu}\right\rangle\right)^{2} .
\end{aligned}
$$

The convexity of the trace distance [106] then gives 
$D_{\operatorname{Tr}}\left(\rho, \sigma_{\alpha}\right)^{2} \leq\left(\sum_{\mu} p_{\alpha, \mu} D_{\operatorname{Tr}}\left(\left|\psi_{\alpha, \mu}\right\rangle,\left|\phi_{\alpha, \mu}\right\rangle\right)\right)^{2} \leq n \epsilon$,

where $\sigma_{\alpha}:=\sum_{\mu} p_{\alpha, \mu}\left|\phi_{\alpha, \mu}\right\rangle\left\langle\phi_{\alpha, \mu}\right|$. Therefore, by choosing sufficiently large $\alpha$, we can find a classical state $\sigma_{\alpha}$ that is arbitrarily close to $\rho$ in trace distance. Hence, $\delta(\rho)=0$. As shown in Ref. [69], this implies that $\rho$ is classical.

Finally, we note that the same conclusions hold for $\hat{\mathcal{W}}_{k}$. Instead of the inequality $\langle N\rangle \leq n \mathcal{V}_{1}$, we use $\langle N\rangle \leq n \mathcal{W}_{1}$.

It is also worth noting that states can be close to classical in trace distance but have arbitrarily large variance. For example, take a superposition of number states $\left|\psi_{l}\right\rangle=$ $\sqrt{1-\epsilon}|0\rangle+\sqrt{\epsilon}|l\rangle$ with $l>2$. Now, $\left|\left\langle 0 \mid \psi_{l}\right\rangle\right|^{2}=1-\epsilon$ but $V\left(\left|\psi_{l}\right\rangle, x\right)=1 / 2+\epsilon l$, which can be made arbitrarily large by choosing large enough $l$.

\section{APPENDIX F: CONVEXITY OF QFI MEASURES}

The QFI of any single observable $A$ is convex: for any ensemble of states $\rho_{\mu}$ with probabilities $p_{\mu}$, $F\left(\sum_{\mu} p_{\mu} \rho_{\mu}, A\right) \leq \sum_{\mu} p_{\mu} F\left(\rho_{\mu}, A\right)$. Then,

$$
\begin{gathered}
\mathcal{F}_{k}\left(\sum_{\mu} p_{\mu} \rho_{\mu}\right) \leq \max _{T: \operatorname{dim}} \sum_{T=k} p_{\mu} \operatorname{Tr}_{T}\left[F\left(\rho_{\mu}\right)-I / 2\right]^{+} \\
\leq \max _{\left\{T_{\mu}: \operatorname{dim} T_{\mu}=k\right\}} \sum_{\mu} p_{\mu} \operatorname{Tr}_{T}\left[F\left(\rho_{\mu}\right)-I / 2\right]^{+} \\
=\sum_{\mu} p_{\mu} \mathcal{F}_{k}\left(\rho_{\mu}\right) .
\end{gathered}
$$

The same applies to $\mathcal{G}_{k}$.

\section{APPENDIX G: NONCLASSICAL STATE NOT WITNESSED BY QFI}

We give an example of a state whose nonclassicality is not witnessed by the QFI measure: $\rho=(1-p) \times$ $\sum_{n=1}^{\infty} p^{n-1}|n\rangle\langle n|$. This was used in a proof [88] of lack of sufficiency of a nonclassicality witness by Vogel [16] and is nonclassical for all $p \in(0,1)$. Using the expression (14), we have

$$
\begin{aligned}
F(\rho, x)= & \left(\frac{1-p}{p}\right) \sum_{n>m \geq 1}^{\infty} \frac{\left(p^{n}-p^{m}\right)^{2}}{p^{n}+p^{m}}|\langle n|x| m\rangle|^{2} \\
& +\left(\frac{1-p}{p}\right) \sum_{m \geq 1}^{\infty} p^{m}|\langle 0|x| m\rangle|^{2} \\
= & \left(\frac{1-p}{p}\right)\left[\sum_{m=1}^{\infty} \frac{\left(p^{m+1}-p^{m}\right)^{2}}{p^{m+1}+p^{m}} \frac{(m+1)}{2}+\frac{p}{2}\right]
\end{aligned}
$$

$$
\begin{gathered}
=\left(\frac{1-p}{2 p}\right)\left[p+\sum_{m=1}^{\infty} p^{m} \frac{(1-p)^{2}}{1+p}(m+1)\right] \\
=\frac{1-p}{2}+\frac{(1-p)^{3}}{2 p(1+p)}\left[\sum_{n=0}^{\infty} n p^{n-1}-1\right] .
\end{gathered}
$$

(Note that this state is symmetric with respect to phase rotations, so $x$ can be any quadrature.) After some algebra, we get

$$
F(\rho, x)=\frac{3}{2}\left(\frac{1-p}{1+p}\right)
$$

and $F(\rho, x)<1 / 2$ for $p>1 / 2$, so the QFI does not detect the nonclassicality of this state.

\section{APPENDIX H: MONOTONICITY PROOFS}

We start by proving monotonicity of $\mathcal{V}_{k}$ for pure states by checking the behavior under each of the elementary operations.

(1) Addition of uncorrelated classical ancilla modes: The covariance matrix for a product of states is simply the direct sum $V\left(|\psi\rangle_{S}|\boldsymbol{\alpha}\rangle_{A}\right)=V\left(|\psi\rangle_{S}\right) \oplus$ $V\left(|\boldsymbol{\alpha}\rangle_{A}\right)$ with $V\left(|\boldsymbol{\alpha}\rangle_{A}\right)=I / 2$. Hence, the vector $\boldsymbol{v}\left(|\psi\rangle_{S}|\boldsymbol{\alpha}\rangle_{A}\right)$ is just $\boldsymbol{v}\left(|\psi\rangle_{S}\right)$ with zeros appended, so $\mathcal{V}_{k}$ is unchanged.

(2) PL unitaries and displacements: The eigenvalues of $V$ are manifestly invariant under these unitaries.

(3) Destructive measurements: We divide the whole set of modes into $S$ and $A$, where $A$ is to be measured. Let $T_{S}, T_{A}$ be their respective (orthogonal) subspaces of the total phase space, such that $T_{S} \oplus T_{A}=$ $\mathbb{R}^{2\left(n_{S}+n_{A}\right)}$. Taking any global state $|\psi\rangle_{S A}$ and $k \leq 2 n_{S}$,

$$
\begin{aligned}
\mathcal{V}_{k}\left(|\psi\rangle_{S A}\right) & =\max _{T: \operatorname{dim} T=k} \operatorname{Tr}_{T}\left[V\left(|\psi\rangle_{S A}\right)-I / 2\right]^{+} \\
& \geq \max _{T \subseteq T_{S}: \operatorname{dim} T=k} \operatorname{Tr}_{T}\left[V\left(|\psi\rangle_{S A}\right)-I / 2\right]^{+} .
\end{aligned}
$$

Now we use a crucial property of the variance [107]: For any observable $x_{S}$ acting only on $S$ and (rank-1) POVM acting only on $A$,

$$
V\left(|\psi\rangle_{S A}, x_{S}\right) \geq \sum_{m} p_{m} V\left(\left|\phi_{m}\right\rangle_{S}, x_{S}\right)
$$

where the $\left|\phi_{m}\right\rangle_{S}$ is the state resulting from measurement outcome $m$ with probability $p_{m}$. This inequality extends to a trace of the covariance matrix over any subspace $T \subseteq T_{S}$, hence, 


$$
\begin{gathered}
\mathcal{V}_{k}\left(|\psi\rangle_{S A}\right) \geq \max _{T \subseteq T_{S}: \operatorname{dim}} \sum_{T=k} p_{m} p_{m} \operatorname{Tr}_{T} \\
{\left[V\left(\left|\phi_{m}\right\rangle_{S}\right)-I / 2\right]^{+} .}
\end{gathered}
$$

For any single $m$, we therefore have

$$
\begin{aligned}
\mathcal{V}_{k}\left(|\psi\rangle_{S A}\right) & \geq p_{m} \max _{T \subseteq T_{S}: \operatorname{dim} T=k} \operatorname{Tr}_{T}\left[V\left(\left|\phi_{m}\right\rangle_{S}\right)-I / 2\right]^{+} \\
& =p_{m} \mathcal{V}_{k}\left(\left|\phi_{m}\right\rangle_{S}\right) .
\end{aligned}
$$

In the case $k=2 n_{S}$, there is no maximization on the right-hand side of Eq. (H3), so

$$
\begin{aligned}
\mathcal{V}_{2 n_{S}}\left(|\psi\rangle_{S A}\right) & \geq \sum_{m} p_{m} \operatorname{Tr}_{T_{S}}\left[V\left(\left|\phi_{m}\right\rangle_{S}\right)-I / 2\right]^{+} \\
& =\sum_{m} p_{m} \mathcal{V}_{2 n_{S}}\left(\left|\phi_{m}\right\rangle_{S}\right) .
\end{aligned}
$$

(4) Classical randomness and coarse graining: In the pure state case, the only such operation allowed is to coarse grain the measurement outcomes that give the same output (otherwise, mixed states are produced) - this changes nothing.

It is easily checked that all of the above logic works identically for $\mathcal{W}_{k}$, the only difference being that maximization is now performed over symplectic subspaces of dimension $2 k$.

For the convex roof $\hat{\mathcal{V}}_{k}$, elements (1) and (2) work as above, so we first address (3). For any $\epsilon>0$, we can find a pure state decomposition $\rho_{S A}=\sum_{\mu} r_{\mu}\left|\psi_{\mu}\right\rangle\left\langle\left.\psi_{\mu}\right|_{S A}\right.$ such that $\sum_{\mu} r_{\mu} \mathcal{V}_{k}\left(\left|\psi_{\mu}\right\rangle_{S A}\right) \leq \hat{\mathcal{V}}_{k}\left(\rho_{S A}\right)+\epsilon$. Let the POVM on $A$ act on $\left|\psi_{\mu}\right\rangle_{S A}$ to give $\left|\phi_{m \mid \mu}\right\rangle_{S}$ with probability $p_{m \mid \mu}$. For any fixed $m$,

$$
\begin{aligned}
& \hat{\mathcal{V}}_{k}\left(\rho_{S A}\right)+\epsilon \geq \sum_{\mu} r_{\mu} p_{m \mid \mu} \mathcal{V}_{k}\left(\left|\phi_{m \mid \mu}\right\rangle_{S}\right) \\
& \geq p_{m} \hat{\mathcal{V}}_{k}\left(\sigma_{S \mid m}\right)
\end{aligned}
$$

where $p_{m} \sigma_{S \mid m}=\sum_{\mu} r_{\mu} p_{m \mid \mu}\left|\phi_{m \mid \mu}\right\rangle\left\langle\left.\phi_{m \mid \mu}\right|_{S}\right.$ is the state obtained from measurement of $\rho_{S A}$ with probability $p_{m}$. Letting $\epsilon \rightarrow 0$ gives the desired result. In the case $k=2 n_{S}$, we have the stronger inequality $\hat{\mathcal{V}}_{2 n_{S}}\left(\rho_{S A}\right) \geq$ $\sum_{m} p_{m} \hat{\mathcal{V}}_{2 n_{S}}\left(\rho_{m S}\right)$.

Monotonicity under coarse graining simply follows from convexity of $\hat{\mathcal{V}}_{k}$.

Again, everything works analogously for $\hat{\mathcal{W}}_{k}$.

We now prove monotonicity for $\mathcal{F}_{k}$. The QFI matrix of a product state is again a direct sum $F\left(\rho_{S} \otimes \rho_{A}\right)=F\left(\rho_{S}\right) \oplus$ $F\left(\rho_{A}\right)$ due to additivity of the QFI [108], with $F\left(\rho_{A}\right) \leq I / 2$ if $\rho_{A}$ is classical. Invariance under PL unitaries is due to the quadratic form $F(\rho, \boldsymbol{r} \cdot \boldsymbol{q})=r^{T} F(\rho) \boldsymbol{r}$. Displacements have the action $q \rightarrow q+$ constant for any quadrature $q$, under which $F(\rho, q)$ is unchanged.
Part (3) hinges on the property of the QFI analogous to Eq. (H2) [74]:

$$
F\left(\rho_{S A}, x_{S}\right) \geq \sum_{m} p_{m} F\left(\sigma_{S \mid m}, x_{S}\right) .
$$

Then, the proofs of monotonicity of $\mathcal{F}_{k}$ and $\mathcal{G}_{k}$ proceed exactly as above.

For special cases where the QFI matrix is the same for every outcome of a measurement, a stronger constraint can be given: the monotonicity of the eigenvalues of the QFI matrix. The proof follows from the CourantFischer theorem [79],

$$
f_{i}=\max _{T: \operatorname{dim}} \min _{T=i \mathbf{r} \in T:|\boldsymbol{r}|=1} \boldsymbol{r}^{T}[F-I / 2]^{+} \boldsymbol{r} .
$$

Following the same logic as above, for any initial state $\rho_{S A}$ and a measurement on $A$, we have

$$
\begin{gathered}
f_{i}\left(\rho_{S A}\right)=\max _{T: \operatorname{dim} T=i \mathbf{r} \in T:|r|=1} \min _{T}\left[F\left(\rho_{S A}, \mathbf{r} \cdot \mathbf{q}\right)-1 / 2\right]^{+} \\
\geq \max _{T \subseteq T_{S}: \operatorname{dim} T=i \mathbf{r} \in T:|r|=1} \min _{|r|}\left[F\left(\rho_{S A}, \mathbf{r} \cdot \mathbf{q}\right)-1 / 2\right]^{+} \\
\geq \max _{T \subseteq T_{S}: \operatorname{dim} T=i} \min _{\mathbf{r} \in T:|r|=1} \sum_{m} p_{m}\left[F\left(\sigma_{S \mid m}, \mathbf{r} \cdot \mathbf{q}\right)-1 / 2\right]^{+} .
\end{gathered}
$$

When all outcomes have the same QFI matrix independent of $m$, for any $m$ we have

$$
\begin{aligned}
f_{i}\left(\rho_{S A}\right) & \geq \max _{T \subseteq T_{S}: \operatorname{dim} T=i} \min _{\mathbf{r} \in T:|\boldsymbol{r}|=1}\left[F\left(\sigma_{S \mid m}, \mathbf{r} \cdot \mathbf{q}\right)-1 / 2\right]^{+} \\
& =f_{i}\left(\sigma_{S \mid m}\right) .
\end{aligned}
$$

This applies both to $\mathcal{P}_{0}$ (since the measurement is trivial) and to Gaussian states with Gaussian measurements (see Appendix J).

The same argument works for $g_{i}$.

\section{APPENDIX I: QFI MATRIX FOR GAUSSIAN STATES}

According to Williamson's theorem [53], every covariance matrix can be diagonalized with a symplectic transformation: $V=S D S^{T}$, where $S \in S p(2 n)$, and $D=\operatorname{diag}\left(d_{1}, d_{1}, d_{2}\right.$, $\left.d_{2}, \ldots, d_{n}, d_{n}\right)$ is the covariance matrix of a product of $n$ thermal states. A straightforward calculation gives the QFI matrix for a thermal state:

$$
V=D \Leftrightarrow F=\frac{1}{4} D^{-1}
$$

From Eq. (14), we see that under a linear transformation $V \rightarrow S V S^{T}$, the QFI matrix transforms in the same way: $F \rightarrow S F S^{T}$. Hence, 


$$
V=S D S^{T} \Leftrightarrow F=\frac{1}{4} S D^{-1} S^{T}
$$

To arrive at the claimed expression, we use the fact that $S \Omega S^{T}=\Omega$, or $S \Omega=\Omega\left(S^{T}\right)^{-1}$ :

$$
\begin{aligned}
\Omega V^{-1} \Omega^{T} & =\Omega\left(S^{T}\right)^{-1} D^{-1} S^{-1} \Omega^{T} \\
& =S \Omega D^{-1} \Omega^{T} S^{T} \\
& =S D^{-1} S \\
& =4 F .
\end{aligned}
$$

\section{APPENDIX J: GAUSSIAN TRANSFORMATIONS}

A crucial property of Gaussian POVMs $E(\boldsymbol{\alpha})=$ $\pi^{-n} D(\boldsymbol{\alpha}) \Lambda D(\boldsymbol{\alpha})^{\dagger}$ is that every outcome has the same covariance matrix independent of the measurement outcome $\boldsymbol{\alpha}$ [53]. Therefore, the same conditions apply to deterministic state transformations as to probabilistic ones. This also implies that it is always sufficient to consider a single measurement step.

\section{Single-mode $\mathcal{P}_{0}$ transformations}

We consider a single system mode $S$ interacting with a single ancilla mode $A$ (which is sufficient by Lemma 1), with covariance matrices $V$ and $Y$, respectively. From Lemma 2, we can assume the PL unitary interaction to consist of a single beam splitter of reflectivity $\eta$, with phase rotations of each mode before and after. Thus, the covariance matrix of the output can be written as

$V^{\prime}=(1-\eta) R_{S} V R_{S}^{T}+\eta R_{A} Y R_{A}^{T}, \quad R_{S}, R_{A} \in K(1)$

We determine the set of $V^{\prime}$ achievable by varying $\eta, R_{S}, R_{A}$. First, consider fixing $\eta$. Given a pair of Hermitian matrices $A, B$, there exists a set of linear inequalities constraining the eigenvalues of their sum $C=A+B$ in terms of the eigenvalues of $A$ and $B$. In the two-dimensional case, three inequalities are necessary and sufficient for the existence of the triple $(A, B, C)$ [109] (Sec. III. 2). Applied to Eq. (J1), these inequalities are

$$
\begin{aligned}
& v_{+}^{\prime} \leq(1-\eta) v_{+}+\eta y_{+}, \\
& v_{-}^{\prime} \leq(1-\eta) v_{+}+\eta y_{-}, \\
& v_{-}^{\prime} \leq(1-\eta) v_{-}+\eta y_{+},
\end{aligned}
$$

where the eigenvalues of $V$ are $v_{+} \geq v_{-}$and similar for $Y, V^{\prime}$. To eliminate $\eta$, we use the fact that $\operatorname{Tr} V^{\prime}=$ $(1-\eta) \operatorname{Tr} V+\eta \operatorname{Tr} Y$, i.e.,

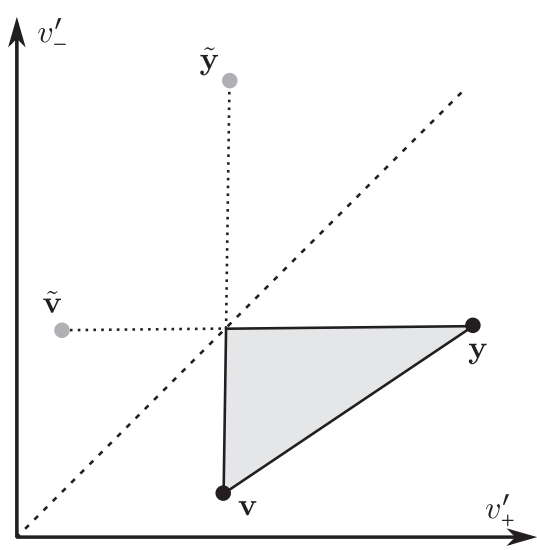

FIG. 6. The region of achievable $\mathbf{v}^{\prime}$ for fixed $\mathbf{v}$ and $\mathbf{y}$ is shaded. The points $\tilde{\mathbf{v}}$ and $\tilde{\mathbf{y}}$ are the reflections of $\mathbf{v}$ and $\mathbf{y}$ in the line $v_{+}^{\prime}=v_{-}^{\prime}$.

$$
v_{+}^{\prime}+v_{-}^{\prime}=(1-\eta)\left(v_{+}+v_{-}\right)+\eta\left(y_{+}+y_{-}\right) .
$$

Thus, we obtain the following necessary and sufficient conditions for the existence of $\eta, R_{S}, R_{A}$ satisfying Eq. (J1):

$$
\begin{aligned}
& v_{+}^{\prime}\left(y_{-}-v_{-}\right) \leq v_{-}^{\prime}\left(y_{+}-v_{+}\right)+v_{+} y_{-}-v_{-} y_{+}, \\
& v_{-}^{\prime}\left(y_{+}-v_{-}\right) \leq v_{+}^{\prime}\left(y_{-}-v_{+}\right)+v_{+} y_{+}-v_{-} y_{-}, \\
& v_{-}^{\prime}\left(y_{-}-v_{+}\right) \leq v_{+}^{\prime}\left(y_{+}-v_{-}\right)+v_{-} y_{-}-v_{+} y_{+} .
\end{aligned}
$$

As illustrated in Fig. 6, these inequalities correspond to a triangular region in the $\left(v_{+}^{\prime}, v_{-}^{\prime}\right)$ plane.

Now we use the ability to choose any classical ancilla, i.e., to choose any $y_{+} \geq y_{-} \geq 1 / 2$. Clearly, every classical $V^{\prime}$ is achievable by choosing any classical $Y$ and swapping the states using $\eta=1$. Given a nonclassical $V$ (meaning $v_{-}<1 / 2$ ), the achievable nonclassical region is as shown in Fig. 3. This can be seen geometrically by considering triangles as in Fig. 6. It is bounded by two constraints: one situation where $y_{+} \rightarrow \infty$, imposing that $v_{-}^{\prime} \geq v_{-}$, the other situation where $y_{+}=y_{-}=1 / 2$. The inequality corresponding to the latter situation is found to be

$$
\frac{1 / 2-v_{-}^{\prime}}{v_{+}^{\prime}-1 / 2} \leq \frac{1 / 2-v_{-}}{v_{+}-1 / 2}
$$

This determines the two necessary and sufficient monotones $\mathcal{N}_{1}, \mathcal{N}_{2}$ for $\mathcal{P}_{0}$ transformations.

\section{Single-mode $\mathcal{G} \mathcal{P}_{\mathbb{N}}$ transformations}

We first demonstrate the necessity of the monotones (assuming a nonclassical initial state). Monotonicity of $\mathcal{N}_{1}$ follows immediately from Theorem 4 since $f_{1}=\left(2 v_{-}\right)^{-1}$. For $\mathcal{N}_{3}$, we first convince ourselves that it suffices to consider a pure ancilla and measurement consisting of projection onto pure Gaussian states, i.e., with POVM 
elements of the form $\pi^{-1} D(\alpha)|s\rangle\langle s| D(\alpha)^{\dagger}$, where $|s\rangle$ is a pure squeezed state. This works because any protocol with more general mixed elements can be obtained by a coarsegrained mixture of such pure cases.

We use the following result about Gaussian measurements at the level of covariance matrices [96-98]: suppose that a two-mode state has covariance matrix written in the $2 \times 2$ block form

$$
\left(\begin{array}{ll}
A & C \\
C^{T} & B
\end{array}\right)
$$

Then projection of the second mode onto a pure Gaussian state with covariance matrix $Z$ results in the first mode having the covariance matrix

$$
A-C(B+Z)^{-1} C^{T} .
$$

In the present case, we start with system and ancilla covariance matrices $V_{S}, V_{A}$. We apply a beam-splitter operation with reflectivity $\eta$ represented by

$$
R=\left(\begin{array}{cc}
\sqrt{1-\eta} I & -\sqrt{\eta} I \\
\sqrt{\eta} I & \sqrt{1-\eta} I
\end{array}\right) .
$$

This results in $R\left(V_{S} \oplus V_{A}\right) R^{T}$; hence, in Eq. (J6) we have $A=(1-\eta) V_{S}+\eta V_{A}, B=\eta V_{S}+(1-\eta) V_{A}, C=$ $\sqrt{\eta(1-\eta)}\left(V_{S}-V_{A}\right)$. The most general pure squeezed state has

$Z=\frac{1}{2}\left(\begin{array}{cc}z^{-1} \cos ^{2} \theta+z \sin ^{2} \theta & \left(z^{-1}-z\right) \cos \theta \sin \theta \\ \left(z^{-1}-z\right) \cos \theta \sin \theta & z^{-1} \sin ^{2} \theta+z \cos ^{2} \theta\end{array}\right)$,

where $z \geq 1$. Also note that, since we take $V_{A}=I / 2$ (corresponding to a coherent state), $V_{S}$ and $V_{A}$ can be simultaneously diagonalized by single-mode phase rotations. Therefore, without loss of generality, we may write

$$
\begin{aligned}
& A=\left(\begin{array}{cc}
(1-\eta) v_{+}+\eta / 2 & 0 \\
0 & (1-\eta) v_{-}+\eta / 2
\end{array}\right), \\
& B=\left(\begin{array}{cc}
\eta v_{+}+(1-\eta) / 2 & 0 \\
0 & \eta v_{-}+(1-\eta) / 2
\end{array}\right), \\
& C=\left(\begin{array}{cc}
\sqrt{\eta(1-\eta)}\left(v_{+}-1 / 2\right) & 0 \\
0 & \sqrt{\eta(1-\eta)}\left(v_{-}-1 / 2\right)
\end{array}\right) .
\end{aligned}
$$

We put these into Eq. (J7) and obtain (complicated) expressions for the eigenvalues $v_{+}^{\prime}, v_{-}^{\prime}$. Evaluating the expression for $\mathcal{N}_{3}$ and performing an optimization over $\theta, \eta$ with all other parameters fixed, we obtain

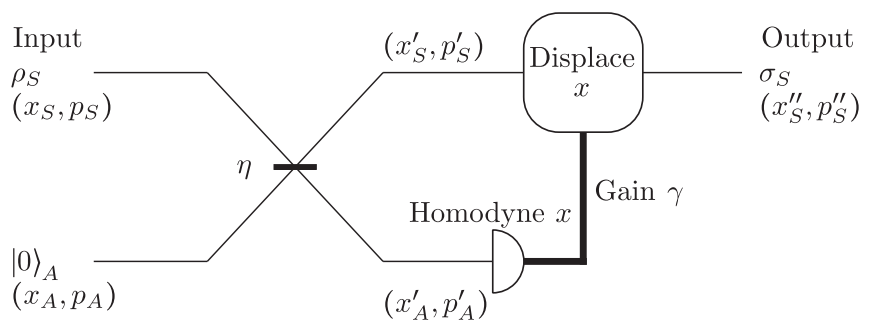

FIG. 7. A $\mathcal{G} \mathcal{P}_{\mathbb{N}}$ operation that achieves a transformation with unchanged $\mathcal{N}_{3}$.

$$
\max _{\theta, \eta} \frac{1 / 2-v_{-}^{\prime}}{2-1 / v_{+}^{\prime}}=\frac{1 / 2-v_{-}}{2-1 / v_{+}}
$$

attained at $\theta=0, \eta=1 / 2$.

For a clearer physical picture and to demonstrate sufficiency, we now give an explicit operation which takes $\rho \mapsto \sigma$ when $\mathcal{N}_{3}(\rho)=\mathcal{N}_{3}(\sigma)$. This operation also includes the conditional displacement on the output that is necessary to achieve a deterministic transformation. As illustrated in Fig. 7, the protocol uses a vacuum ancilla, a beam splitter with reflectivity $\eta$ plus homodyne detection, and feed forward to a displacement with gain factor $\gamma$. The homodyne is performed on the most noisy quadrature.

We choose $x_{S}, p_{S}$ such that $V\left(\rho_{S}, x_{S}\right)=v_{+}$, $V\left(\rho_{S}, p_{S}\right)=v_{-}$and perform a homodyne measurement of $x_{A}^{\prime}=\sqrt{1-\eta} x_{A}-\sqrt{\eta} x_{S}$. Let the final displaced quadratures be $x_{S}^{\prime \prime}=x_{S}^{\prime}+\gamma x_{A}^{\prime}, p_{S}^{\prime \prime}=p_{S}^{\prime}$. We calculate the final variances as

$$
v_{-}^{\prime}=V\left(\sigma_{S}, p_{S}^{\prime \prime}\right)=(1-\eta) v_{-}+\eta / 2
$$

and $v_{+}^{\prime}=V\left(\sigma_{S}, x_{S}^{\prime \prime}\right)$. Using

$$
\begin{aligned}
x_{S}^{\prime \prime} & =\left(\sqrt{1-\eta} x_{S}+\sqrt{\eta} x_{A}\right)+\gamma\left(-\sqrt{\eta} x_{S}+\sqrt{1-\eta} x_{A}\right) \\
& =(\sqrt{1-\eta}-\gamma \sqrt{\eta}) x_{S}+(\sqrt{\eta}+\gamma \sqrt{1-\eta}) x_{A},
\end{aligned}
$$

and noting that $x_{S}$ and $x_{A}$ are initially uncorrelated, we have

$$
\begin{aligned}
v_{+}^{\prime}= & V\left(\sigma_{S}, x_{S}^{\prime \prime}\right)=(\sqrt{1-\eta}-\gamma \sqrt{\eta})^{2} V\left(\rho_{S}, x_{S}\right) \\
& +(\sqrt{\eta}+\gamma \sqrt{1-\eta})^{2} V\left(\rho_{A}, x_{A}\right) \\
= & (\sqrt{1-\eta}-\gamma \sqrt{\eta})^{2} v_{+}+(\sqrt{\eta}+\gamma \sqrt{1-\eta})^{2} \times \frac{1}{2} .
\end{aligned}
$$

This expression is minimized by choosing a gain factor

$$
\gamma=\frac{\sqrt{\eta(1-\eta)}\left(v_{+}-1 / 2\right)}{\eta v_{+}+(1-\eta) \times 1 / 2},
$$

from which one finds 


$$
v_{+}^{\prime}=\frac{v_{+} / 2}{\eta v_{+}+(1-\eta) \times 1 / 2}=\left(\frac{1-\eta}{v_{+}}+2 \eta\right)^{-1} .
$$

From this and Eq. (J13), we have

$$
\frac{2-1 / v_{+}^{\prime}}{2-1 / v_{+}}=1-\eta=\frac{1 / 2-v_{-}^{\prime}}{1 / 2-v_{-}}
$$

showing that $\mathcal{N}_{3}$ is unchanged. Moreover, by choosing appropriate $\eta$, we see that $v_{+}^{\prime}$ can be varied in the interval $\left[1 / 2, v_{+}\right]$. Hence, any point lying on the curve $\mathcal{N}_{3}=$ constant with $v_{-}^{\prime} \geq v_{-}$is reachable.

Finally, we can see that any point lying above the curves $\mathcal{N}_{1}, \mathcal{N}_{3}=$ constant is reachable. After the above operation, one simply adds classical noise to $v_{-}^{\prime}$ without affecting $v_{+}^{\prime}$. In Fig. 3, this corresponds to moving vertically upward in the plane.

[1] S. Lloyd and S. L. Braunstein, Quantum Computation over Continuous Variables, Phys. Rev. Lett. 82, 1784 (1999).

[2] N. C. Menicucci, P. van Loock, M. Gu, C. Weedbrook, T.C. Ralph, and M.A. Nielsen, Universal Quantum Computation with Continuous-Variable Cluster States, Phys. Rev. Lett. 97, 110501 (2006).

[3] M. Gu, C. Weedbrook, N. C. Menicucci, T. C. Ralph, and P. van Loock, Quantum Computing with ContinuousVariable Clusters, Phys. Rev. A 79, 062318 (2009).

[4] A. P. Lund, A. Laing, S. Rahimi-Keshari, T. Rudolph, J. L. O'Brien, and T. C. Ralph, Boson Sampling from a Gaussian State, Phys. Rev. Lett. 113, 100502 (2014).

[5] N. Gisin and R. Thew, Quantum Communication, Nat. Photonics 1, 165 (2007).

[6] T. C. Ralph, Continuous Variable Quantum Cryptography, Phys. Rev. A 61, 010303 (1999).

[7] A. M. Lance, T. Symul, W. P. Bowen, B. C. Sanders, T. Tyc, T. C. Ralph, and P. K. Lam, Continuous-Variable Quantum-State Sharing via Quantum Disentanglement, Phys. Rev. A 71, 033814 (2005).

[8] M. G. A. Paris, Quantum Estimation For Quantum Technology, Int. J. Quantum. Inform. 07, 125 (2009).

[9] D. Šafránek, A.R. Lee, and I. Fuentes, Quantum Parameter Estimation Using Multi-Mode Gaussian States, New J. Phys. 17, 073016 (2015).

[10] S. Yokoyama, R. Ukai, S. C. Armstrong, C. Sornphiphatphong, T. Kaji, S. Suzuki, J.-. Yoshikawa, H. Yonezawa, N. C. Menicucci, and A. Furusawa, UltraLarge-Scale Continuous-Variable Cluster States Multiplexed in the Time Domain, Nat. Photonics 7, 982 (2013).

[11] R. J. Glauber, The Quantum Theory of Optical Coherence, Phys. Rev. 130, 2529 (1963).

[12] E. C. G. Sudarshan, Equivalence of Semiclassical and Quantum Mechanical Descriptions of Statistical Light Beams, Phys. Rev. Lett. 10, 277 (1963).

[13] L. Mandel, Non-Classical States of the Electromagnetic Field, Phys. Scr. T12, 34 (1986).

[14] M. S. Kim, W. Son, V. Bužek, and P. L. Knight, Entanglement by a Beam Splitter: Nonclassicality as a
Prerequisite for Entanglement, Phys. Rev. A 65, 032323 (2002).

[15] X.-b. Wang, Theorem for the Beam-Splitter Entangler, Phys. Rev. A 66, 024303 (2002).

[16] W. Vogel, Nonclassical States: An Observable Criterion, Phys. Rev. Lett. 84, 1849 (2000).

[17] T. Richter and W. Vogel, Nonclassicality of Quantum States: A Hierarchy of Observable Conditions, Phys. Rev. Lett. 89, 283601 (2002).

[18] E. Shchukin, T. Richter, and W. Vogel, Nonclassicality Criteria in Terms of Moments, Phys. Rev. A 71, 011802 (2005).

[19] M. Hillery, Nonclassical Distance in Quantum Optics, Phys. Rev. A 35, 725 (1987).

[20] C. T. Lee, Measure of the Nonclassicality of Nonclassical States, Phys. Rev. A 44, R2775 (1991).

[21] P. Marian, T. A. Marian, and H. Scutaru, Quantifying Nonclassicality of One-Mode Gaussian States of the Radiation Field, Phys. Rev. Lett. 88, 153601 (2002).

[22] J. K. Asbóth, J. Calsamiglia, and H. Ritsch, Computable Measure of Nonclassicality for Light, Phys. Rev. Lett. 94, 173602 (2005).

[23] C. Gehrke, J. Sperling, and W. Vogel, Quantification of Nonclassicality, Phys. Rev. A 86, 052118 (2012).

[24] W. Vogel and J. Sperling, Unified Quantification of Nonclassicality and Entanglement, Phys. Rev. A 89, 052302 (2014).

[25] S. Ryl, J. Sperling, and W. Vogel, Quantifying Nonclassicality by Characteristic Functions, Phys. Rev. A 95, 053825 (2017).

[26] I. I. Arkhipov, J. Peřina, J. Svozilík, and A. Miranowicz, Nonclassicality Invariant of General Two-Mode Gaussian States, Sci. Rep. 6, 26523 (2016).

[27] B. Coecke, T. Fritz, and R. W. Spekkens, A Mathematical Theory of Resources, Inf. Comput. 250, 59 (2016).

[28] V. Vedral, M. B. Plenio, M. A. Rippin, and P. L. Knight, Quantifying Entanglement, Phys. Rev. Lett. 78, 2275 (1997).

[29] R. Horodecki, P. Horodecki, M. Horodecki, and K. Horodecki, Quantum Entanglement, Rev. Mod. Phys. 81, 865 (2009).

[30] S. D. Bartlett, T. Rudolph, and R. W. Spekkens, Reference Frames, Superselection Rules, and Quantum Information, Rev. Mod. Phys. 79, 555 (2007).

[31] M. Horodecki and J. Oppenheim, Fundamental Limitations for Quantum and Nanoscale Thermodynamics, Nat. Commun. 4, 2059 (2013).

[32] G. Gour, M. P. Müller, V. Narasimhachar, R. W. Spekkens, and N. Y. Halpern, The Resource Theory of Informational Nonequilibrium in Thermodynamics, Phys. Rep. 583, 1 (2015).

[33] N. Ng and M.P. Woods, Resource Theory of Quantum Thermodynamics: Thermal Operations and Second Laws, arXiv:1805.09564.

[34] M. Lostaglio, Thermodynamic Laws for Populations and Quantum Coherence: A Self-Contained Introduction to the Resource Theory Approach to Thermodynamics, arXiv:1807.11549.

[35] J. Åberg, Quantifying Superposition, arXiv:quant-ph/ 0612146. 
[36] T. Baumgratz, M. Cramer,, and M. B. Plenio, Quantifying Coherence, Phys. Rev. Lett. 113, 140401 (2014).

[37] A. Streltsov, G. Adesso, and M. B. Plenio, Colloquium: Quantum Coherence as a Resource, Rev. Mod. Phys. 89, 041003 (2017).

[38] A. Grudka, K. Horodecki, M. Horodecki, P. Horodecki, R. Horodecki, P. Joshi, W. Kłobus, and A. Wójcik, Quantifying Contextuality, Phys. Rev. Lett. 112, 120401 (2014).

[39] R. Gallego and L. Aolita, Resource Theory of Steering, Phys. Rev. X 5, 041008 (2015).

[40] M. G. Genoni and M. G. A. Paris, Quantifying NonGaussianity for Quantum Information, Phys. Rev. A 82, 052341 (2010).

[41] R. Takagi and Q. Zhuang, Convex Resource Theory of Non-Gaussianity, arXiv:1804.04669.

[42] Q. Zhuang, P. W. Shor, and J. H. Shapiro, Resource Theory of Non-Gaussian Operations, arXiv:1803.07580.

[43] F. Albarelli, M. G. Genoni, M. G. A. Paris, and A. Ferraro, Resource Theory of Quantum Non-Gaussianity and Wigner Negativity, arXiv:1804.05763.

[44] S. L. Braunstein, Squeezing as an Irreducible Resource, Phys. Rev. A 71, 055801 (2005).

[45] R. Filip, Distillation of Quantum Squeezing, Phys. Rev. A 88, 063837 (2013).

[46] S. Rahimi-Keshari, T. Kiesel, W. Vogel, S. Grandi, A. Zavatta, and M. Bellini, Quantum Process Nonclassicality, Phys. Rev. Lett. 110, 160401 (2013).

[47] K. K. Sabapathy, Quantum-Optical Channels that Output Only Classical States, Phys. Rev. A 92, 052301 (2015).

[48] K. K. Sabapathy, Process Output Nonclassicality and Nonclassicality Depth of Quantum-Optical Channels, Phys. Rev. A 93, 042103 (2016).

[49] F. Albarelli, A. Ferraro, M. Paternostro, and M. G. A. Paris, Nonlinearity as a Resource for Nonclassicality in Anharmonic Systems, Phys. Rev. A 93, 032112 (2016).

[50] M. Idel, D. Lercher, and M. M. Wolf, An Operational Measure for Squeezing, J. Phys. A 49, 445304 (2016).

[51] M. Idel and R. Koenig, On Quantum Additive Gaussian Noise Channels, Quantum Inf. Comput. 17, 0283 (2017).

[52] K. C. Tan, T. Volkoff, H. Kwon, and H. Jeong, Quantifying the Coherence between Coherent States, Phys. Rev. Lett. 119, 190405 (2017).

[53] C. Weedbrook, S. Pirandola, R. García-Patrón, N. J. Cerf, T. C. Ralph, J. H. Shapiro, and S. Lloyd, Gaussian Quantum Information, Rev. Mod. Phys. 84, 621 (2012).

[54] M. Reck, A. Zeilinger, H. J. Bernstein, and P. Bertani, Experimental Realization of Any Discrete Unitary Operator, Phys. Rev. Lett. 73, 58 (1994).

[55] P. Kok, W. J. Munro, K. Nemoto, T. C. Ralph, J. P. Dowling, and G. J. Milburn, Linear Optical Quantum Computing with Photonic Qubits, Rev. Mod. Phys. 79, 135 (2007).

[56] M. G. A. Paris, Displacement Operator by Beam Splitter, Phys. Lett. A 217, 78 (1996).

[57] P. Kok, H. Lee, and J. P. Dowling, Single-Photon Quantum-Nondemolition Detectors Constructed with Linear Optics and Projective Measurements, Phys. Rev. A 66, 063814 (2002).
[58] U. L. Andersen, To. Gehring, C. Marquardt, and G. Leuchs, 30 Years of Squeezed Light Generation, Phys. Scr. 91, 053001 (2016).

[59] E. Chitambar and G. Gour, Critical Examination of Incoherent Operations and a Physically Consistent Resource Theory of Quantum Coherence, Phys. Rev. Lett. 117, 030401 (2016).

[60] B. Yadin, J. Ma, D. Girolami, M. Gu, and V. Vedral, Quantum Processes Which Do Not Use Coherence, Phys. Rev. X 6, 041028 (2016).

[61] J. I. de Vicente and A. Streltsov, Genuine Quantum Coherence, J. Phys. A 50, 045301 (2017).

[62] E. B. Davies and J. T. Lewis, An Operational Approach to Quantum Probability, Commun. Math. Phys. 17, 239 (1970).

[63] N. Killoran, F. E. S. Steinhoff, and M. B. Plenio, Converting Nonclassicality into Entanglement, Phys. Rev. Lett. 116, 080402 (2016).

[64] J. Wenger, R. Tualle-Brouri, and P. Grangier, NonGaussian Statistics from Individual Pulses of Squeezed Light, Phys. Rev. Lett. 92, 153601 (2004).

[65] V. Parigi, A. Zavatta, M. Kim, and M. Bellini, Probing Quantum Commutation Rules by Addition and Subtraction of Single Photons to/from a Light Field, Science 317, 1890 (2007).

[66] C. M. Caves, Quantum Limits on Noise in Linear Amplifiers, Phys. Rev. D 26, 1817 (1982).

[67] T. C. Ralph, A. P. Lund, and A. Lvovsky, Nondeterministic Noiseless Linear Amplification of Quantum Systems, AIP Conf. Proc. 1110, 155 (2009).

[68] A. Zavatta, J. Fiurášek, and M. Bellini, A High-Fidelity Noiseless Amplifier for Quantum Light States, Nat. Photonics 5, 52 (2010).

[69] R. Nair, Nonclassical Distance in Multimode Bosonic Systems, Phys. Rev. A 95, 063835 (2017).

[70] J. M. C. Malbouisson and B. Baseia, On the Measure of Nonclassicality of Field States, Phys. Scr. 67, 93 (2003).

[71] A. J. Leggett, Testing the Limits of Quantum Mechanics: Motivation, State of Play, Prospects, J. Phys. Condens. Matter 14, R415 (2002).

[72] F. Fröwis, P. Sekatski, W. Dür, N. Gisin, and N. Sangouard, Macroscopic Quantum States: Measures, Fragility and Implementations, Rev. Mod. Phys. 90, 025004 (2018).

[73] E. Oudot, P. Sekatski, F. Fröwis, N. Gisin, and N. Sangouard, Two-Mode Squeezed States as Schrödinger Cat-like States, J. Opt. Soc. Am. B 32, 2190 (2015).

[74] B. Yadin and V. Vedral, General Framework for Quantum Macroscopicity in Terms of Coherence, Phys. Rev. A 93, 022122 (2016).

[75] H. P. Robertson, The Uncertainty Principle, Phys. Rev. 34, 163 (1929).

[76] D. Stoler, Equivalence Classes of Minimum Uncertainty Packets, Phys. Rev. D 1, 3217 (1970).

[77] M. Hillery, Total Noise and Nonclassical States, Phys. Rev. A 39, 2994 (1989).

[78] C. W. Lee and H. Jeong, Quantification of Macroscopic Quantum Superpositions within Phase Space, Phys. Rev. Lett. 106, 220401 (2011). 
[79] R. A. Horn and C. R. Johnson, Matrix Analysis (Cambridge University Press, Cambridge, England, 1985), Chap. 4, pp. 692-692.

[80] M. de Gosson, Symplectic Geometry and Quantum Mechanics (Birkhäuser Basel, Basel, 2006).

[81] G. Vidal, Entanglement of Pure States for a Single Copy, Phys. Rev. Lett. 83, 1046 (1999).

[82] G. Tóth and D. Petz, Extremal Properties of the Variance and the Quantum Fisher Information, Phys. Rev. A 87, 032324 (2013).

[83] S. Yu, Quantum Fisher Information as the Convex Roof of Variance, arXiv:1302.5311.

[84] Á. Rivas and A. Luis, Precision Quantum Metrology and Nonclassicality in Linear and Nonlinear Detection Schemes, Phys. Rev. Lett. 105, 010403 (2010).

[85] F. Fröwis, P. Sekatski, and W. Dür, Detecting Large Quantum Fisher Information with Finite Measurement Precision, Phys. Rev. Lett. 116, 090801 (2016).

[86] F. Fröwis, Lower Bounds on the Size of General Schrödinger-Cat States from Experimental Data, J. Phys. A 50, 114003 (2017).

[87] D. Girolami and B. Yadin, Witnessing Multipartite Entanglement by Detecting Asymmetry, Entropy 19, 124 (2017).

[88] L. Diósi, Comment on "Nonclassical States: An Observable Criterion”, Phys. Rev. Lett. 85, 2841 (2000).

[89] G. Vidal and R.F. Werner, Computable Measure of Entanglement, Phys. Rev. A 65, 032314 (2002).

[90] K. Życzkowski, P. Horodecki, A. Sanpera, and M. Lewenstein, Volume of the Set of Separable States, Phys. Rev. A 58, 883 (1998).

[91] A. P. Lund, H. Jeong, T. C. Ralph, and M. S. Kim, Conditional Production of Superpositions of Coherent States with Inefficient Photon Detection, Phys. Rev. A $\mathbf{7 0}$, 020101 (2004).

[92] M. Szczykulska, T. Baumgratz, and A. Datta, MultiParameter Quantum Metrology, Adv. Phys. X 1, 621 (2016).

[93] G. Adesso, S. Ragy, and Antony R. Lee, Continuous Variable Quantum Information: Gaussian States and Beyond, Open Syst. Inf. Dyn. 21, 1440001 (2014).

[94] A. Ferraro, S. Olivares, and M. G. A. Paris, Gaussian States in Continuous Variable Quantum Information, arXiv:quant-ph/0503237.
[95] R. Simon, N. Mukunda, and B. Dutta, QuantumNoise Matrix for Multimode Systems: $U(n)$ Invariance, Squeezing, and Normal Forms, Phys. Rev. A 49, 1567 (1994).

[96] J. Eisert, S. Scheel, and M. B. Plenio, Distilling Gaussian States with Gaussian Operations is Impossible, Phys. Rev. Lett. 89, 137903 (2002).

[97] J. Fiurášek, Gaussian Transformations and Distillation of Entangled Gaussian States, Phys. Rev. Lett. 89, 137904 (2002).

[98] G. Giedke and J. I. Cirac, Characterization of Gaussian Operations and Distillation of Gaussian States, Phys. Rev. A 66, 032316 (2002).

[99] L. Lami, B. Regula, X. Wang, R. Nichols, A. Winter, and G. Adesso, Gaussian Quantum Resource Theories, Phys. Rev. A 98, 022335 (2018).

[100] G. Giedke, J. Eisert, J. I. Cirac, and M. B. Plenio, Entanglement Transformations of Pure Gaussian States, Quantum Inf. Comput. 3, 211 (2003).

[101] F. Fröwis and W. Dür, Measures of Macroscopicity for Quantum Spin Systems, New J. Phys. 14, 093039 (2012).

[102] H. Kwon, K. C. Tan, T. Volkoff, and H. Jeong, Nonclassicality of Light as a Quantifiable Resource for Quantum Metrology, arXiv:1804.09355.

[103] E. Chitambar, D. Leung, L. Mančinska, .aris Ozols, and A. Winter, Everything You Always Wanted to Know About LOCC (But Were Afraid to Ask), Commun. Math. Phys. 328, 303 (2014).

[104] C. C. Paige and M. Wei, History and Generality of the CS Decomposition, Linear Algebra Appl. 208-209, 303 (1994).

[105] M. A. de Gosson, The Symplectic Egg, arXiv:1208.5969.

[106] M. A. Nielsen and I. L. Chuang, Quantum Computation and Quantum Information (Cambridge University Press, Cambridge, England, 2010), Chap. 9.

[107] N. Schuch, F. Verstraete, and J. I. Cirac, Quantum Entanglement Theory in the Presence of Superselection Rules, Phys. Rev. A 70, 042310 (2004).

[108] G. Tóth and I. Apellaniz, Quantum Metrology from a Quantum Information Science Perspective, J. Phys. A 47, 424006 (2014).

[109] R. Bhatia, Matrix Analysis, Graduate Texts in Mathematics (Springer, New York, 1997), Vol. 169. 